Preprint typeset in JHEP style - HYPER VERSION

FSU-HEP-080822, LPSC 08-133

\title{
Prospects for Yukawa Unified $S O(10)$ SUSY GUTs at the CERN LHC
}

\author{
Howard Baer $^{a}$, Sabine $\mathrm{Kraml}^{b}$, Sezen Sekmen ${ }^{c}$ and Heaya Summy ${ }^{a, d}$ \\ ${ }^{a}$ Dept. of Physics and Astronomy, University of Oklahoma, Norman, OK 73019, USA \\ ${ }^{b}$ Laboratoire de Physique Subatomique et de Cosmologie, UJF Grenoble 1, \\ CNRS/IN2P3, INPG, 53 Avenue des Martyrs, F-38026 Grenoble, France \\ ${ }^{c}$ Department of Physics, Middle East Technical Univ., TR-06531 Ankara, Turkey \\ ${ }^{d}$ Department of Physics, Florida State University, Tallahassee, FL 32306, USA \\ E-mail: baer@nhn.ou.edu, sabine.kraml@lpsc.in2p3.fr, sezen.sekmen@cern.ch, \\ heaya@hep.fsu.edu
}

\begin{abstract}
The requirement of $t-b-\tau$ Yukawa coupling unification is common in simple grand unified models based on the gauge group $S O(10)$, and it also places a severe constraint on the expected spectrum of superpartners. For Yukawa-unified models with $\mu>0$, the spectrum is characterized by three mass scales: $i$ ). first and second generation scalars in the multi-TeV range, $i i$ ). third generation scalars, $\mu$ and $m_{A}$ in the few-TeV range and iii). gluinos in the $\sim 350-500 \mathrm{GeV}$ range with chargino masses around $100-160$ $\mathrm{GeV}$. In such a scenario, gluino pair production should occur at large rates at the CERN LHC, followed by gluino three-body decays into neutralinos or charginos. Discovery of Yukawa-unified SUSY at the LHC should hence be possible with only $1 \mathrm{fb}^{-1}$ of integrated luminosity, by tagging multi-jet events with $2-3$ isolated leptons, without relying on missing $E_{T}$. A characteristic dilepton mass edge should easily be apparent above Standard Model background. Combining dileptons with $b$-jets, along with the gluino pair production cross section information, should allow for gluino and neutralino mass reconstruction. A secondary corroborative signal should be visible at higher integrated luminosity in the $\tilde{\chi}_{1}^{ \pm} \tilde{\chi}_{2}^{0} \rightarrow 3 \ell$ channel, and should exhibit the same dilepton mass edge as in the gluino cascade decay signal.
\end{abstract}

Keywords: Supersymmetry Phenomenology, Supersymmetric Standard Model, Dark Matter. 


\section{Introduction}

Models for new physics based on $S O(10)$ grand unification and supersymmetry are especially compelling[1]. $S O(10)$ grand unified theories (GUTs) allow for-in addition to gauge coupling unification- matter unification in the sense that all fields of a single generation of the Standard Model (SM) are embedded into the 16-dimensional spinor representation of $S O(10)$. Since there are only 15 fields in a SM generation, the 16th element is occupied by a right-handed neutrino (RHN) field. The RHN is necessary for implementing the see-saw mechanism[2] of generating neutrino masses. In addition, since the gauge group $S O(10)$ is anomaly free, the ad-hoc but fortuitous triangle anomaly cancellation in the SM and in $S U(5)$ GUTs is explained. The addition of supersymmetry to the model allows for a stabilization of the weak scale-GUT scale hierarchy, and is supported by the fact that the measured weak scale gauge couplings actually unify at $Q=M_{G U T} \simeq 2 \times 10^{16} \mathrm{GeV}$ under minimal supersymmetric standard model (MSSM) renormalization group evolution (RGE). Along with gauge and matter unification, the simplest $S O(10)$ SUSY GUT models may also have Yukawa coupling unification, especially for the third generation.

On the minus side, $S O(10)$ SUSY GUT models in 4 dimensions are often unwieldy, due in part to the large Higgs representations which are needed to break the GUT gauge symmetry. In recent years, progress has been made in constructing SUSY GUT theories in 5 or 6 spacetime dimensions [3]. In these cases, the GUT symmetry can be broken by extra-dimensional compactification on for instance an orbifold. Such theories maintain many of the essential predictions of SUSY GUTs, while dispensing with GUT-breaking via large Higgs representations. In any case, if we consider that the MSSM arises from superstring theory as a 4-d effective theory below $M_{G U T}$, then 6-7 extra dimensions will have to be compactified, and the description of string $\rightarrow$ GUT $\rightarrow$ MSSM is likely to be more complicated than any of the existing models.

To avoid dealing with the unknown physics above the GUT scale, in this paper we will assume that an $S O(10)$ SUSY GUT model breaks (either via the Higgs mechanism or via compactification of extra dimensions) to the SM gauge group at energy scale $Q=M_{G U T}$. Below $M_{G U T}$, we assume the MSSM is the correct effective field theory which describes nature. We will further assume that the superpotential above $M_{G U T}$ is of the form

$$
\hat{f} \ni f \hat{\psi}_{16} \hat{\psi}_{16} \hat{\phi}_{10}+\cdots
$$

so that the three third generation Yukawa couplings $f_{t}, f_{b}$ and $f_{\tau}$ are unified at $M_{G U T} \cdot{ }^{1} \mathrm{We}$ will further make the reasonable assumption that the GUT scale soft SUSY breaking terms are constrained by the $S O(10)$ gauge symmetry such that matter scalars have a common mass $m_{16}$, Higgs scalars have a common mass $m_{10}$ and there is a common trilinear soft parameter $A_{0}$. The bilinear soft term $B$ can be traded as usual for $\tan \beta$, the ratio of Higgs field vevs, while the magnitude of the superpotential Higgs mass $\mu$ is determined in terms

\footnotetext{
${ }^{1}$ It is simple in this context to include as well the effect of a third generation neutrino Yukawa coupling $f_{\nu}$; this effect has been shown to be small, although it can affect Yukawa coupling unification by a few per cent, if the right hand neutrino mass scale is within a few orders of magnitude of $M_{G U T}$ 纯.
} 
of $M_{Z}^{2}$ via the electroweak symmetry breaking minimization conditions. Here, electroweak symmetry is broken radiatively (REWSB) due to the large top quark mass.

In order to accomodate REWSB, it is well-known that the GUT scale Higgs soft masses must be split such that $m_{H_{u}}^{2}<m_{H_{d}}^{2}$, in order to fulfill the EWSB minimization conditions. Two possibilities- full $D$-term splitting applied to all scalar masses (DT), or splitting applied only to Higgs soft terms (HS) - have been considered 5, 40. The latter HS case was found to yield a higher degree of Yukawa coupling unification when $m_{16} \sim 10$ $\mathrm{TeV}$, so that this is the case we will consider here. We parametrize the Higgs splitting as $m_{H_{u, d}}^{2}=m_{10}^{2} \mp 2 M_{D}^{2}$. The Higgs splitting can originate via a large near-GUT-scale threshold correction arising from the neutrino Yukawa coupling: see Ref. [5] for discussion. Thus, the Yukawa unified SUSY model is determined by the parameter space

$$
m_{16}, m_{10}, M_{D}^{2}, m_{1 / 2}, A_{0}, \tan \beta, \operatorname{sign}(\mu)
$$

along with the top quark mass, which we take to be $m_{t}=171 \mathrm{GeV} .^{2}$

In previous work, the above parameter space was scanned over (via random scans [0, 4] and also by more efficient Markov Chain Monte Carlo (MCMC) scans 8) to search for Yukawa unified solutions. The quantity

$$
R=\frac{\max \left(f_{t}, f_{b}, f_{\tau}\right)}{\min \left(f_{t}, f_{b}, f_{\tau}\right)}
$$

was examined, where solutions with $R \simeq 1$ gave apparent Yukawa coupling unification. For superpotential Higgs mass parameter $\mu>0$ (as favored by $(g-2)_{\mu}$ measurements), Yukawa unified solutions with $R \sim 1$ were found but only for special choices of GUT scale boundary conditions $[0,0,5,4,10,8]$ :

$$
A_{0} \sim-2 m_{16}, m_{10} \sim 1.2 m_{16},
$$

with $m_{1 / 2} \ll m_{16}$ and $\tan \beta \sim 50$. In fact, models with this sort of boundary conditions were derived even earlier in the context of inverted scalar mass hierarchy models (IMH) which attempt to reconcile suppression of flavor-changing and $C P$-violating processes with naturalness via multi-TeV first/second generation and sub- $\mathrm{TeV}$ scale third generation scalars 11]. The Yukawa-unified spectral solutions were thus found in Refs. [4, 8] to occur with the above peculiar choice of boundary conditions along with $m_{16} \sim 3-20$ $\mathrm{TeV}$ and $m_{1 / 2} \sim 50-100 \mathrm{GeV}$. For accurate spectrum evaluation, we adopted the Isajet 7.75 algorithm 12, 13, which includes

- full two-loop RG evolution of both couplings and soft SUSY breaking (SSB) terms 14,

- minimization of the RG-improved one-loop scalar potential at an optimized scale $M_{S U S Y}$ (we take $M_{S U S Y}=\sqrt{m_{\tilde{t}_{L}} m_{\tilde{t}_{R}}}$, which accounts for leading two-loop terms [15],

- implementation of complete 1-loop $t, b$ and $\tau$ threshold corrections 16, 17 and

- implementation of complete 1-loop corrections for all sparticle masses 17.

\footnotetext{
${ }^{2}$ Recent measurements from CDF and D0[6] now find a central value for $m_{t}$ of $172.6 \pm 1.4 \mathrm{GeV}$.
} 
- a hybrid approach of changing gauge and Yukawa coupling beta functions as different soft terms are integrated out of the effective theory [13].

A plot of Yukawa coupling evolution for case A of Table 1 of Ref. [8] with $m_{16} \simeq 9 \mathrm{TeV}$ is shown in Fig. 1. Here, we clearly see the importance of the MSSM theshold corrections, which give rise to the discontinuities located around $Q=M_{S U S Y} \sim 3 \mathrm{TeV}$.

Based on the above work [ [ [], the sparticle mass spectra from Yukawa-unified SUSY models are characterized by the following conditions:

- first and second generation scalars have masses in the $3-20 \mathrm{TeV}$ regime,

- third generation scalars, $\mu$ and $m_{A}$ have masses in the $1-4 \mathrm{TeV}$ regime (owing to the inverted scalar mass hierarchy),

- gluinos $\tilde{g}$ have mass in the $350-500 \mathrm{GeV}$ range,

- light charginos have mass $m_{\tilde{\chi}_{1}} \sim 100-160 \mathrm{GeV}$ and

- the lightest neutralino $\tilde{\chi}_{1}^{0}$ is nearly pure bino with mass $m_{\tilde{\chi}_{1}^{0}} \simeq 50-80 \mathrm{GeV}$.

The presence of a bino-like $\tilde{\chi}_{1}^{0}$ along with multi-TeV scalars gives rise to a neutralino dark matter $(\mathrm{DM})$ relic abundance that is typically in the range $\Omega_{\tilde{\chi}_{1}^{0}} h^{2} \sim 10^{2}-10^{5}$, i.e. far above [18] the WMAP measured [19] value $\Omega_{C D M} h^{2}=0.111_{-0.015}^{+0.011}(2 \sigma)$ by several orders of magnitude.

A very compelling way out for solutions with $m_{16} \sim 5-20 \mathrm{TeV}$, is to not assume that the lightest neutralino $\tilde{\chi}_{1}^{0}$ is the lightest SUSY particle (LSP). Instead, we assume that the axino $\tilde{a}$ (the spin- $\frac{1}{2}$ element of the supermultiplet containing the axion) is in fact

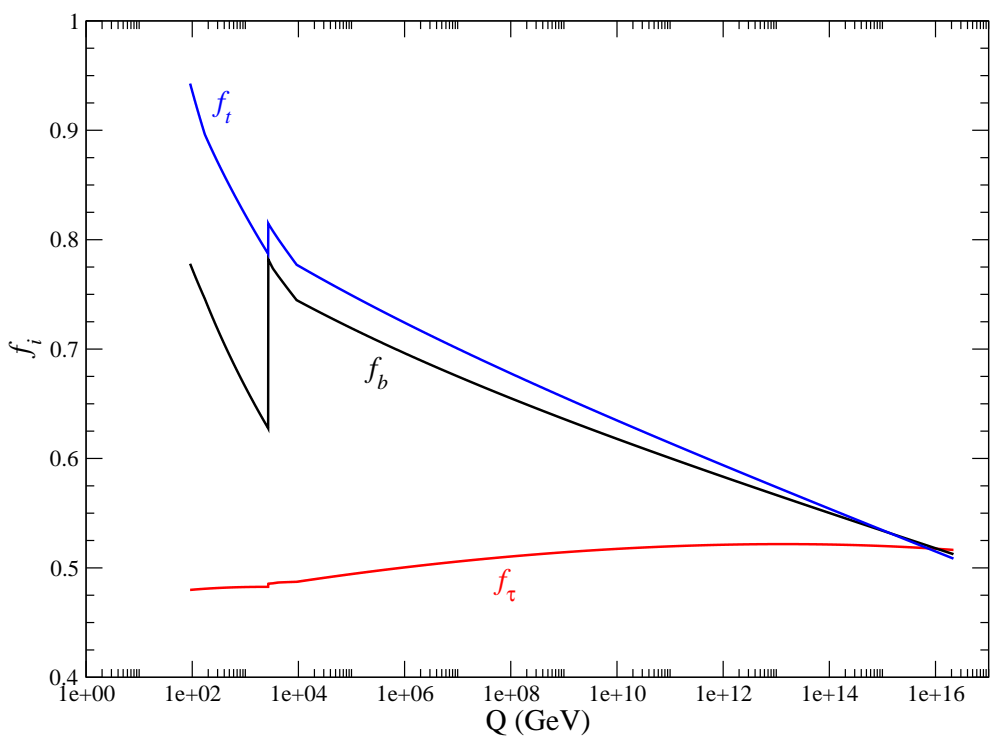

Figure 1: Plot of $f_{t}, f_{b}$ and $f_{\tau}$ evolution from the weak scale to the GUT scale for Point A of Table 1. The large jumps around $3 \mathrm{TeV}$ correspond to the MSSM threshold corrections. 
the LSP 20]. The axino mass is very model-dependent, but can be anywhere in the keV$\mathrm{GeV}$ range[21, 20]. In this case, the neutralino mass over-abundance is greatly reduced since $\tilde{\chi}_{1}^{0} \rightarrow \tilde{a} \gamma$ decay can occur with a lifetime of order 0.03 sec. This decay time is sufficiently short that late-time neutralino decay to axino in the early universe should not upset successful predictions of Big Bang Nucleosynthesis (BBN). The relic abundance then gets reduced by the ratio $m_{\tilde{a}} / m_{\tilde{\chi}_{1}^{0}}$ which can be of order $10^{-2}-10^{-5}$. The $\tilde{a}$ coming from $\tilde{\chi}_{1}^{0}$ decay would actually constitute warm dark matter[22]. However, axinos can also be produced thermally in the early universe as cold dark matter 20, 23]. Thus, in the axino LSP scenario, we would actually have dominantly thermally produced cold axino DM with a small admixture of warm axino DM arising from $\tilde{\chi}_{1}^{0} \rightarrow \tilde{a} \gamma$ decays. In addition, there is also the possibility of a significant presence of axion CDM.

In fact, in this class of Yukawa-unified solutions with $m_{16}$ in the multi-TeV range, we also expect the gravitino $\tilde{G}$ to lie in the multi-TeV range. The cosmological gravitino problem- wherein gravitinos produced thermally in the early universe suffer a late-time decay, thus destroying the successful predictions of BBN- can be avoided. For $m_{\tilde{G}} \lesssim 5$ $\mathrm{TeV}$, the re-heat temperature $T_{R}$ must be $T_{R} \lesssim 10^{5} \mathrm{GeV}$ [24], thus creating tension with most viable mechanisms for baryogenesis 25]. However, for $m_{\tilde{G}} \gtrsim 5 \mathrm{TeV}$, the re-heat bound is much higher: $T_{R} \lesssim 10^{8}-10^{9} \mathrm{GeV}$. This range of $T_{R}$ is exactly what is needed for baryogenesis via non-thermal leptogenesis [26], wherein the heavy right-hand neutrino states are not produced thermally, but rather via inflaton decay. It also turns out to be the exact range needed to generate a dominantly cold axino DM universe. Thus, the whole scenario fits together to offer a consistent cosmological picture of BBN, baryogenesis and cold DM composed of axinos 27]!

An alternative but equally compelling solution to the CDM problem in Yukawa-unified SUSY models occurs in cases where $m_{16}$ is as low as the $\sim 3 \mathrm{TeV}$ range. In this case, the $\tilde{\chi}_{1}^{0}$ can remain as the LSP. Since $m_{16}$ is so low, Yukawa coupling unification only occurs at the $R \sim 1.09$ level. However, $\tilde{\chi}_{1}^{0} \tilde{\chi}_{1}^{0}$ can now annihilate through the light Higgs $h$ resonance at a sufficient rate to obtain the desired relic density with $\tilde{\chi}_{1}^{0}$ remaining as the LSP [8].

In this paper, we explore the consequences of Yukawa-unified SUSY models for sparticle detection at the LHC. We focus most of our attention on two cases presented in Table 1 of Ref. [8]: 1. point A with $m_{16} \sim 9 \mathrm{GeV}$ and an axino LSP, and 2. point D with $m_{16} \sim 3 \mathrm{TeV}$ and a neutralino LSP. While our studies here focus on just two cases, we maintain that the qualitative features of all Yukawa-unified SUSY LHC signatures should be rather similar to these two cases. In fact, the collider phenomenology of these cases is rather similar between the two, since in the first case the neutralino decays to an axino far beyond the detector boundaries. Thus, in both cases the lightest neutralino $\tilde{\chi}_{1}^{0}$ leads to missing $E_{T}$ at collider experiments.

For the benefit of the reader, we present in Table 1 the two case studies we examine. We present the parameter space values, sparticle mass spectrum, and in addition the total treelevel LHC sparticle production cross section. We also list as percentages some contributing $2 \rightarrow 2$ subprocess reactions.

The remainder of this paper is organized as follows. In Sec. 2, we present some details 


\begin{tabular}{lcc}
\hline parameter & Pt. A & Pt. D \\
\hline$m_{16}$ & 9202.9 & 2976.5 \\
$m_{1 / 2}$ & 62.5 & 107.0 \\
$A_{0}$ & -19964.5 & -6060.3 \\
$m_{10}$ & 10966.1 & 3787.9 \\
$\tan \beta$ & 49.1 & 49.05 \\
$M_{D}$ & 3504.4 & 1020.8 \\
$f_{t}$ & 0.51 & 0.48 \\
$f_{b}$ & 0.51 & 0.47 \\
$f_{\tau}$ & 0.52 & 0.52 \\
$\mu$ & 4179.8 & 331.0 \\
$m_{\tilde{g}}$ & 395.6 & 387.7 \\
$m_{\tilde{u}_{L}}$ & 9185.4 & 2970.8 \\
$m_{\tilde{t}_{1}}$ & 2315.1 & 434.5 \\
$m_{\tilde{b}_{1}}$ & 2723.1 & 849.3 \\
$m_{\tilde{e}_{L}}$ & 9131.9 & 2955.8 \\
$m_{\tilde{\chi}_{1}^{ \pm}}$ & 128.8 & 105.7 \\
$m_{\tilde{\chi}_{2}^{0}}$ & 128.6 & 105.1 \\
$m_{\tilde{\chi}_{1}^{0}}$ & 55.6 & 52.6 \\
$m_{A}$ & 3273.6 & 776.8 \\
$m_{h}$ & 125.4 & 111.1 \\
\hline$\sigma[\mathrm{fb}]$ & 75579.1 & 89666.1 \\
$\%_{(\tilde{g}}(\tilde{g})$ & 86.8 & 80.5 \\
$\%_{(}\left(\tilde{\chi}_{1}^{ \pm} \tilde{\chi}_{2}^{0}\right)$ & 8.8 & 12.8 \\
$\%_{(}\left(\tilde{t}_{1} \tilde{t}_{1}\right)$ & 0 & 1.1 \\
\hline & & \\
\hline & &
\end{tabular}

Table 1: Masses and parameters in GeV units for two cases studies points A and D of Ref. [8] using Isajet 7.75 with $m_{t}=171.0 \mathrm{GeV}$. We also list the total tree level sparticle production cross section in $\mathrm{fb}$ at the LHC, plus the percent for several two-body final states.

involving total sparticle production rates at the LHC and also sparticle branching fractions expected in Yukawa-unified SUSY models. In Sec. 3, we generate simulated LHC collider events associated with these two scenarios along with SM background (BG) rates. Since the collider signals are quite similar for both of points A and D, we mainly present figures for just point A. Our main findings include: the Yukawa-unified SUSY scenarios should be easily discoverable at LHC with about $1 \mathrm{fb}^{-1}$ of integrated luminosity, without using missing $E_{T}$ cuts, by requiring events with a high isolated lepton multiplicity: $n_{\ell} \geq 2$ or 3 . In Sec. 4, we present several distributions which can be used to gain information on sparticle masses, especially $m_{\tilde{g}}, m_{\tilde{\chi}_{2}^{0}}$ and $m_{\tilde{\chi}_{1}^{0}}$. The gluino mass $m_{\tilde{g}}$ can be extracted from total cross section results. In Sec. 5, we discuss a corroborating signal in the $p p \rightarrow \tilde{\chi}_{1}^{ \pm} \tilde{\chi}_{2}^{0} \rightarrow 3 \ell+E_{T}^{\text {miss }}$ channel. This channel comes from the soft component of signal events involving direct production of the lighter charginos and neutralinos. Our conclusions are presented in Sec. 6 . 


\section{Cross sections and branching fractions for sparticles in Yukawa-unified models}

Given the characteristic spectrum of superpartners obtained in Yukawa-unified SUSY models, it is useful to examine what sort of new physics signals we would expect at the LHC. Obviously, first/second generation squarks and sleptons in the multi-TeV mass range will essentially decouple from LHC physics. Gluinos- in the $350-500 \mathrm{GeV}$ range- will be produced in abundance via $q \bar{q}$ and $g g$ fusion subprocesses. Charginos and neutralinos, being in the 100-160 GeV range, may also be produced with observable cross sections.

As noted above, we list the tree-level total sparticle production cross sections obtained from Isajet for cases A and D in Table 11. In case A, we find $\sigma(t o t) \sim 8 \times 10^{4} \mathrm{fb}$, so that 8000 sparticle pair events are expected at LHC with just $0.1 \mathrm{fb}^{-1}$ of integrated luminosity. Of this total, $86.7 \%$ comes from gluino pair production, while $8.8 \%$ comes from $\tilde{\chi}_{1} \tilde{\chi}_{2}^{0}$ production and $4.5 \%$ comes from $\tilde{\chi}_{1}^{+} \tilde{\chi}_{1}^{-}$production. In case $\mathrm{D}, \sigma(t o t) \sim 9.6 \times 10^{4} \mathrm{fb}$, with $80.4 \%$ from $\tilde{g} \tilde{g}$ production, $12.8 \%$ from $\tilde{\chi}_{1} \tilde{\chi}_{2}^{0}$ production, $6.4 \%$ from $\tilde{\chi}_{1}^{+} \tilde{\chi}_{1}^{-}$production while top-squark pair production yields just $1.1 \%$ of the total. Given these production cross sections, we expect Yukawa-unified SUSY to yield primarily $\tilde{g} \tilde{g}$ events at the LHC. Gluino pair production typically leads to events with hard jets, hard $E_{T}^{\text {miss }}$ and isolated leptons from the gluino cascade decays[28]. We also expect a soft component coming from $\tilde{\chi}_{1}^{+} \tilde{\chi}_{1}^{-}$and $\tilde{\chi}_{1}^{ \pm} \tilde{\chi}_{2}^{0}$ production. While both these reactions lead to events with rather soft jets, leptons and $E_{T}^{\text {miss }}$, the latter reaction can also yield clean trilepton events 29], which should be visible at LHC above SM backgrounds.

For the case of gluino masses other than those listed in Table 1, we show in Fig. 2 the total gluino pair production rate versus $m_{\tilde{g}}$ at the LHC at tree level (solid) and nextto-leading-order (NLO) using the Prospino program [30]. The scale choice is taken to be $Q=m_{\tilde{g}}$. We take $m_{\tilde{q}}$ to be $3 \mathrm{TeV}$ (blue) and $9 \mathrm{TeV}$ (red). As can be seen, the results hardly vary between this range of squark masses. The tree level results agree well with Isajet, but the NLO results typically show an enhancement by a factor $\sim 1.6$. Thus, we expect Yukawa-unified SUSY models to yield $p p \rightarrow \tilde{g} \tilde{g} X$ events at a 30-150 pb level at LHC.

In Fig. 3, we show the total -ino pair production cross sections versus chargino mass $m_{\tilde{\chi}_{1}^{ \pm}}$. While $\tilde{\chi}_{1}^{ \pm} \tilde{\chi}_{2}^{0}$ and $\tilde{\chi}_{1}^{+} \tilde{\chi}_{1}^{-}$production dominate, and have rates around $10^{3}-10^{4} \mathrm{fb}$ over the range of interest, there exists a sub-dominant rate for $\tilde{\chi}_{1}^{ \pm} \tilde{\chi}_{1}^{0}$ and also $\tilde{\chi}_{1}^{0} \tilde{\chi}_{2}^{0}$ production.

Now that we see that Yukawa-unified SUSY will yield dominantly gluino pair production events at the LHC, we next turn to the gluino branching fractions in order to understand their event signatures. All sparticle branching fractions are calculated with Isajet 7.75. In Fig. 4, we show various gluino branching fractions for points A and D. We see immediately that in both cases, $B F\left(\tilde{g} \rightarrow b \bar{b} \tilde{\chi}_{2}^{0}\right)$ dominates at around $56 \%$. This is followed by $B F\left(\tilde{g} \rightarrow b \bar{b} \tilde{\chi}_{1}^{0}\right)$ at $\sim 16 \%$, and $B F\left(\tilde{g} \rightarrow b \bar{t} \tilde{\chi}_{1}^{+}\right)$and $B F\left(\tilde{g} \rightarrow t \bar{b} \tilde{\chi}_{1}^{-}\right)$each at $\sim 10 \%$. Decays to first and second generation quarks are much suppressed due to the large first and second generation squark masses. From these results, we expect gluino pair production events to be rich in $b$-jets, $E_{T}^{\text {miss }}$ and occassional isolated leptons from the leptonic decays $\tilde{\chi}_{1} \rightarrow \ell \bar{\nu}_{\ell} \tilde{\chi}_{1}^{0}$ and $\tilde{\chi}_{2}^{0} \rightarrow \ell^{+} \ell^{-} \tilde{\chi}_{1}^{0}$, where $\ell=e$ or $\mu$. 


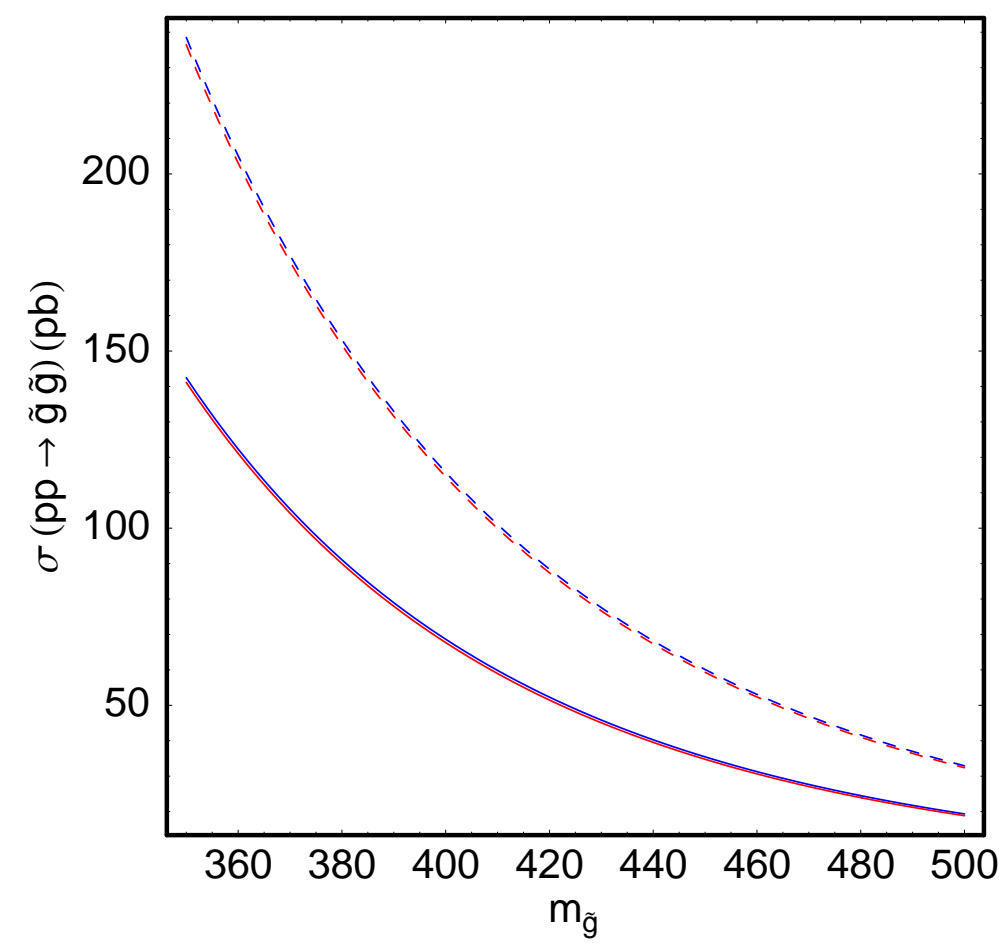

Figure 2: Plot of $\sigma(p p \rightarrow \tilde{g} \tilde{g} X)$ in pb at $\sqrt{s}=14 \mathrm{TeV}$ versus $m_{\tilde{g}}$. We use Prospino with scale choice $Q=m_{\tilde{g}}$, and show LO (solid) and NLO (dashes) predictions in the vicinity of point A (red) and point D (blue) from Table 1 .

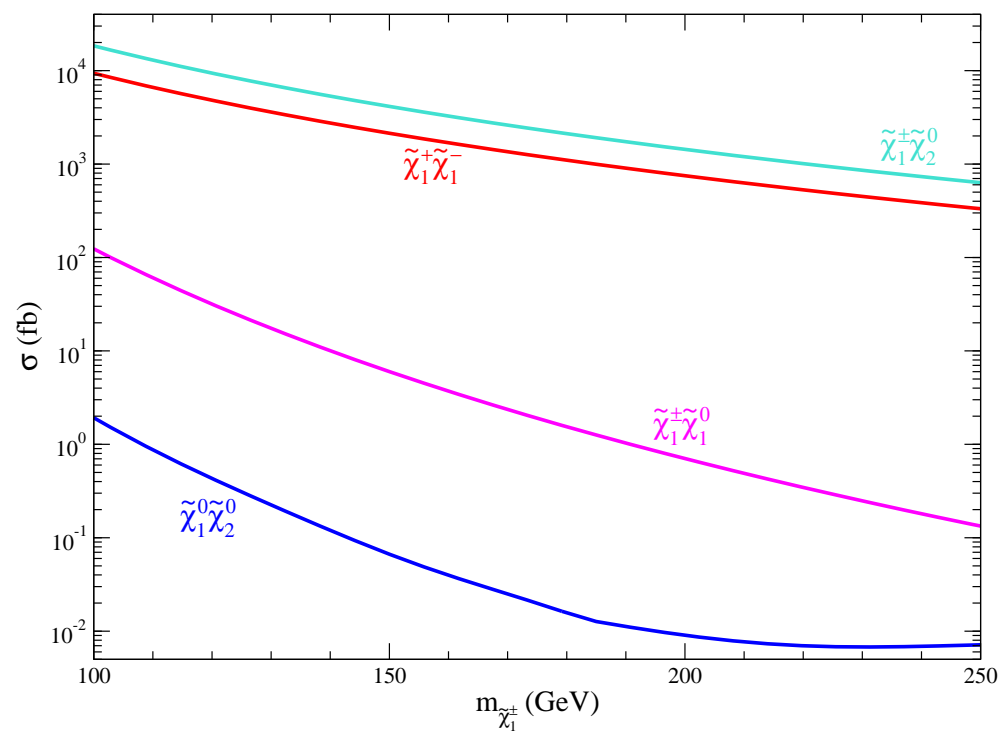

Figure 3: Plot of various -ino pair production processes in fb at $\sqrt{s}=14 \mathrm{TeV}$ versus $m_{\tilde{\chi}_{1}^{ \pm}}$, for $m_{\tilde{q}}=3 \mathrm{TeV}$ and $\mu=m_{\tilde{g}}$, with $\tan \beta=49$ and $\mu>0$. 
Point A Point D

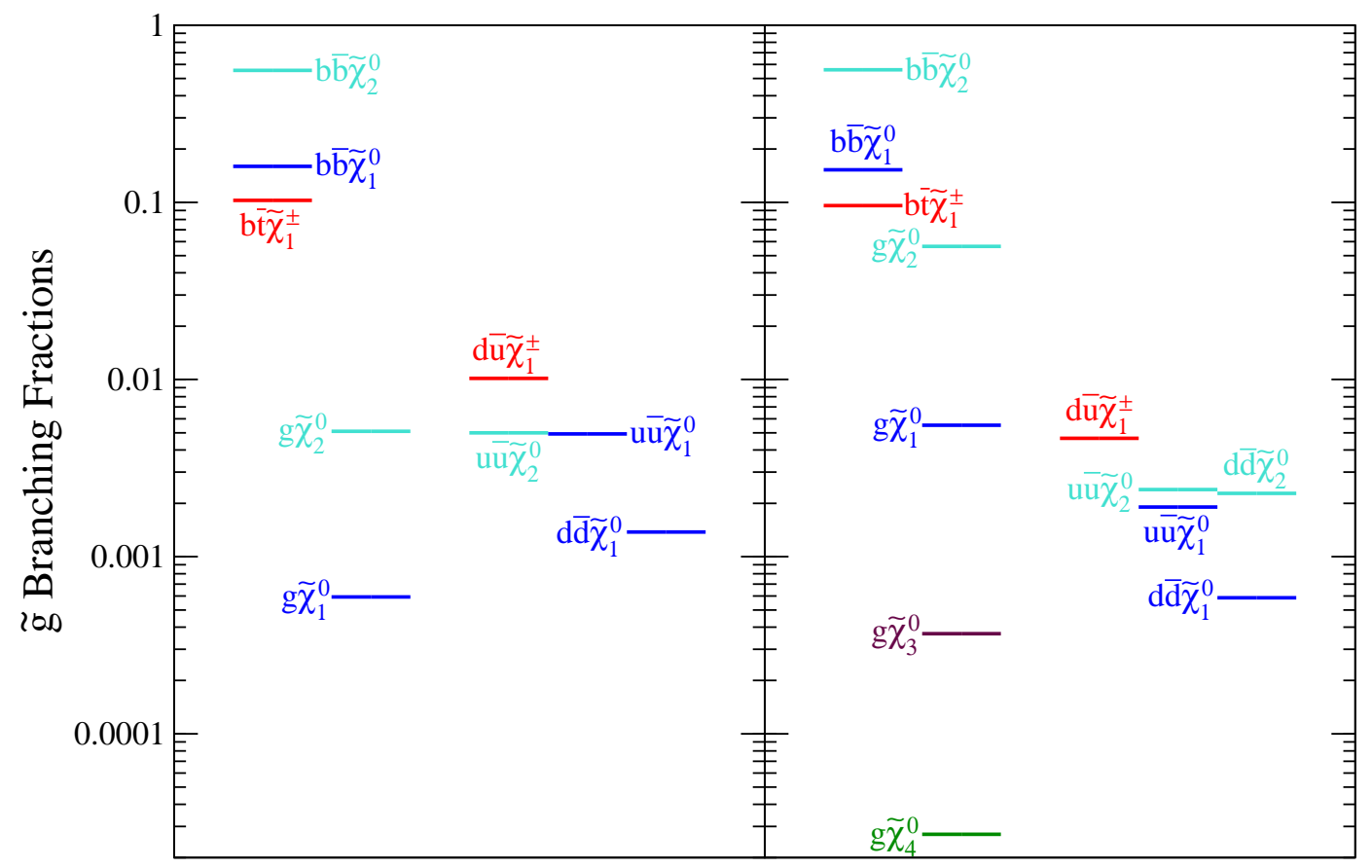

Figure 4: Plot of various sparticle branching fractions taken from Isajet for points A and D from Table 1 .

\section{Gluino pair production signals at the LHC}

To examine collider signals from Yukawa-unified SUSY at the LHC in more detail, we generate $10^{6}$ sparticle pair production events for points $\mathrm{A}$ and $\mathrm{D}$, corresponding to 13 and $11 \mathrm{fb}^{-1}$ of integrated luminosities. We use Isajet 7.75 12] for the simulation of signal and background events at the LHC. A toy detector simulation is employed with calorimeter cell size $\Delta \eta \times \Delta \phi=0.05 \times 0.05$ and $-5<\eta<5$. The HCAL energy resolution is taken to be $80 \% / \sqrt{E}+3 \%$ for $|\eta|<2.6$ and FCAL is $100 \% / \sqrt{E}+5 \%$ for $|\eta|>2.6$. The ECAL energy resolution is assumed to be $3 \% / \sqrt{E}+0.5 \%$. We use a UA1-like jet finding algorithm with jet cone size $R=0.4$ and require that $E_{T}($ jet $)>50 \mathrm{GeV}$ and $|\eta(j e t)|<3.0$. Leptons are considered isolated if they have $p_{T}(e$ or $\mu)>20 \mathrm{GeV}$ and $|\eta|<2.5$ with visible activity within a cone of $\Delta R<0.2$ of $\Sigma E_{T}^{\text {cells }}<5 \mathrm{GeV}$. The strict isolation criterion helps reduce multi-lepton backgrounds from heavy quark $(c \bar{c}$ and $b \bar{b})$ production. We also invoke a lepton identification efficiency of $75 \%$ for leptons with $20 \mathrm{GeV}<p_{T}(\ell)<50 \mathrm{GeV}$, and $85 \%$ for leptons with $p_{T}(\ell)>50 \mathrm{GeV}$.

We identify a hadronic cluster with $E_{T}>50 \mathrm{GeV}$ and $|\eta(j)|<1.5$ as a $b$-jet if it contains a $B$ hadron with $p_{T}(B)>15 \mathrm{GeV}$ and $|\eta(B)|<3$ within a cone of $\Delta R<0.5$ about the jet axis. We adopt a $b$-jet tagging efficiency of $60 \%$, and assume that light quark and gluon jets can be mis-tagged as $b$-jets with a probability $1 / 150$ for $E_{T} \leq 100 \mathrm{GeV}$, $1 / 50$ for $E_{T} \geq 250 \mathrm{GeV}$, with a linear interpolation for $100 \mathrm{GeV}<E_{T}<250 \mathrm{GeV}$ 31]. 


\begin{tabular}{|c|c|c|c|c|}
\hline process & events & $\sigma(\mathrm{fb})$ & $C 1^{\prime}$ & $C 1^{\prime}+E_{T}^{\text {miss }}$ \\
\hline $\mathrm{QCD}\left(p_{T}: 0.05-0.1 \mathrm{TeV}\right)$ & $10^{6}$ & $2.6 \times 10^{10}$ & $4.1 \times 10^{5}$ & - \\
\hline $\mathrm{QCD}\left(p_{T}: 0.1-0.2 \mathrm{TeV}\right)$ & $10^{6}$ & $1.5 \times 10^{9}$ & $1.4 \times 10^{7}$ & - \\
\hline $\mathrm{QCD}\left(p_{T}: 0.2-0.4 \mathrm{TeV}\right)$ & $10^{6}$ & $7.3 \times 10^{7}$ & $6.5 \times 10^{6}$ & 2199 \\
\hline $\mathrm{QCD}\left(p_{T}: 0.4-1.0 \mathrm{TeV}\right)$ & $10^{6}$ & $2.7 \times 10^{6}$ & $2.8 \times 10^{5}$ & 1157 \\
\hline $\mathrm{QCD}\left(p_{T}: 1-2.4 \mathrm{TeV}\right)$ & $10^{6}$ & $1.5 \times 10^{4}$ & 1082 & 25 \\
\hline$W \rightarrow \ell \nu_{\ell}+j e t s$ & $5 \times 10^{5}$ & $3.9 \times 10^{5}$ & 3850 & 1275 \\
\hline$Z \rightarrow \tau \bar{\tau}+j e t s$ & $5 \times 10^{5}$ & $1.4 \times 10^{5}$ & 1358 & 652 \\
\hline$t \bar{t}$ & $3 \times 10^{6}$ & $4.9 \times 10^{5}$ & $8.2 \times 10^{4}$ & 2873 \\
\hline$W W, Z Z, W Z$ & $5 \times 10^{5}$ & $8.0 \times 10^{4}$ & 197 & 7 \\
\hline Total BG & $9.5 \times 10^{6}$ & $2.76 \times 10^{10}$ & $2.13 \times 10^{7}$ & 8188 \\
\hline Point A: & $10^{6}$ & $7.6 \times 10^{4}$ & $3.6 \times 10^{4}$ & 8914 \\
\hline$S / B \rightarrow$ & - & - & 0.002 & 1.09 \\
\hline$S / \sqrt{S+B}\left(1 \mathrm{fb}^{-1}\right) \rightarrow$ & - & - & - & 68 \\
\hline Point D: & $10^{6}$ & $9.0 \times 10^{4}$ & $3.7 \times 10^{4}$ & 10843 \\
\hline$S / B \rightarrow$ & - & - & 0.002 & 1.32 \\
\hline$S / \sqrt{S+B}\left(1 \mathrm{fb}^{-1}\right) \rightarrow$ & - & - & - & 78 \\
\hline
\end{tabular}

Table 2: Events generated and cross sections (in fb) for various SM background and signal processes before and after cuts. The $C 1^{\prime}$ and $E_{T}^{\text {miss }}$ cuts are specified in the text. The $W+j e t s$ and $Z+j e t s$ background has been computed within the restriction $p_{T}(W, Z)>100 \mathrm{GeV}$.

In addition to signal, we have generated background events using Isajet for QCD jet production (jet-types include $g, u, d, s, c$ and $b$ quarks) over five $p_{T}$ ranges as shown in Table 2. Additional jets are generated via parton showering from the initial and final state hard scattering subprocesses. We have also generated backgrounds in the $W+$ jets, $Z+j e t s, t \bar{t}$ (with $m_{t}=171 \mathrm{GeV}$ ) and $W W, W Z, Z Z$ channels at the rates shown in Table 2. The $W+$ jets and $Z+$ jets backgrounds use exact matrix elements for one parton emission, but rely on the parton shower for subsequent emissions.

First we require modest cuts: $n($ jets $) \geq 4$. Also, SUSY events are expected to spray large $E_{T}$ throughout the calorimeter, while QCD dijet events are expected to be typically back-to-back. Thus, we expect QCD background to be peaked at transverse sphericity $S_{T} \sim 0$, while SUSY events have larger values of $S_{T} .{ }^{3}$ The actual $S_{T}$ distribution for point $\mathrm{A}$ is shown in Fig. (the $S_{T}$ distribution for point $\mathrm{D}$ is practically the same). Motivated by this, we require $S_{T}>0.2$ to reject QCD-like events.

We plot the jet $E_{T}$ distributions of the four highest $E_{T}$ jets from Pt. A (in color) and the total SM background (gray histogram) in Fig. 6, ordered from highest to lowest $E_{T}$,

\footnotetext{
${ }^{3}$ Here, $S_{T}$ is the usual sphericity variable, restricted to the transverse plane, as is appropriate for hadron colliders. Sphericity matrix is given as

$$
S=\left(\begin{array}{cc}
\sum p_{x}^{2} & \sum p_{x} p_{y} \\
\sum p_{x} p_{y} & \sum p_{y}^{2}
\end{array}\right)
$$

from which $S_{T}$ is defined as $2 \lambda_{1} /\left(\lambda_{1}+\lambda_{2}\right)$, where $\lambda_{1,2}$ are the larger and smaller eigenvalues of $S$.
} 
with jets labelled as $j 1-j 4$. The histograms are normalized to unity in order to clearly see the differences in distribution shapes. Again, the distributions for point D look very similar. We find that the highest $E_{T}$ jet distribution peaks around $E_{T} \sim 150 \mathrm{GeV}$ with a long tail extending to higher $E_{T}$ values, while for the background it peaks at a lower value of $E_{T} \sim 100 \mathrm{GeV}$. Jet 2 and jet 3 have peak distributions around $100 \mathrm{GeV}$ both for the signal and backgrounds, while the jet 4 distribution backs up against the minimum jet $E_{T}$ requirement that $E_{T}(j e t)>50 \mathrm{GeV}$. Thus, at little cost to signal but with large BG rejection, we require $E_{T}(j 1)>100 \mathrm{GeV}$.

The collection of cuts so far is dubbed $C 1^{\prime}$ [32]:

\section{C1' cuts:}

$$
\begin{aligned}
n(\text { jets }) & \geq 4, \\
E_{T}(j 1, j 2, j 3, j 4) & \geq 100,50,50,50 \mathrm{GeV}, \\
S_{T} & \geq 0.2 .
\end{aligned}
$$

The classic signature for SUSY collider events is the presence of jets plus large $E_{T}^{\text {miss }}$ [33]. In Fig. 0, we show the expected distribution of $E_{T}^{\text {miss }}$ from points $\mathrm{A}$ and D, along with SM BG. We do see that signal becomes comparable to BG around $E_{T}^{\text {miss }} \sim 150 \mathrm{GeV}$. We list cross sections from the two signal cases plus SM backgrounds in Table 2 after cuts $C 1^{\prime}$ plus $E_{T}^{\text {miss }}>150 \mathrm{GeV}$. While signal $S$ is somewhat higher than the summed BG $B$, the signal and $\mathrm{BG}$ rates are rather comparable in this case: $S / B=1.09$ for pt. A while $S / B=1.32$ for pt. D.

Even so, it has been noted in Ref. [32] that $E_{T}^{\text {miss }}$ may be a difficult variable to reliably construct during the early stages of LHC running. The reason is that missing transverse

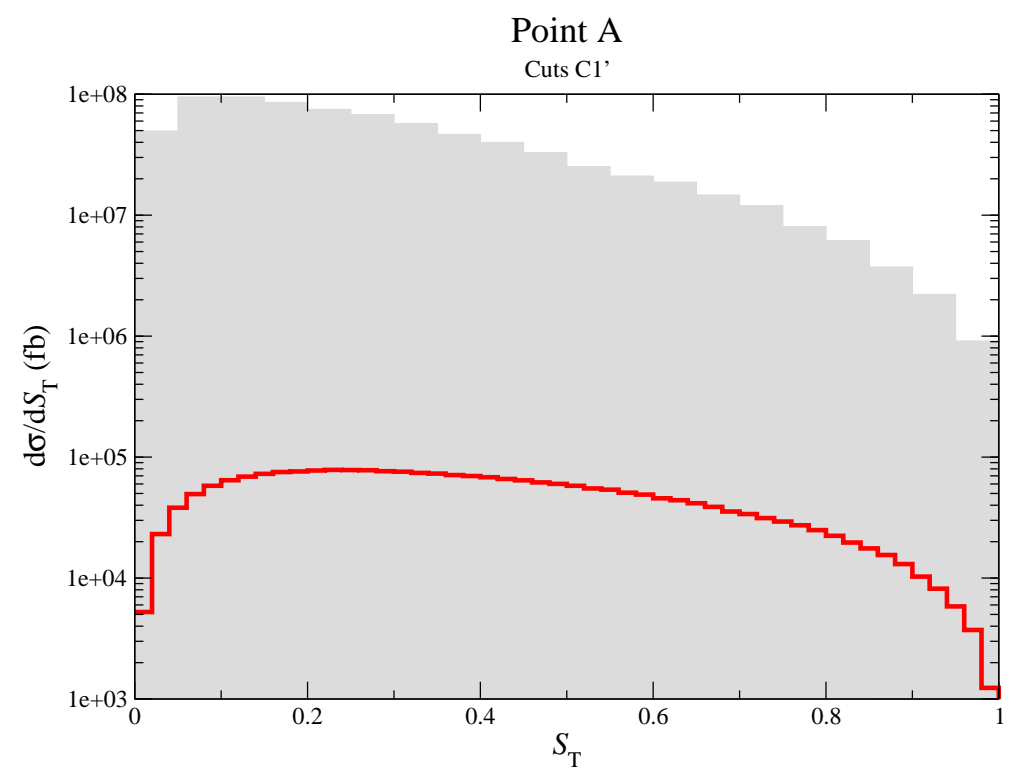

Figure 5: Plot of distribution in transverse sphericity $S_{T}$ for events with cuts $C 1^{\prime}$ from benchmark point A and the summed SM background; point D leads to practically the same distribution. 
energy can arise not only from the presence of weakly interacting neutral particles such as neutrinos or the lightest neutralinos, but also from a variety of other sources, including:

- energy loss from cracks and un-instrumented regions of the detector,

- energy loss from dead cells,

- hot cells in the calorimeter that report an energy deposition even if there is not one,

- mis-measurement in the electromagnetic calorimeters, hadronic calorimeters or muon detectors,

- real missing transverse energy produced in jets due to semi-leptonic decays of heavy flavors,

- muons and

- the presence of mis-identified cosmic rays in events.

Thus, in order to have a solid grasp of expected $E_{T}^{\text {miss }}$ from SM background processes, it will be necessary to have detailed knowledge of the complete detector performance. As experience from the Tevatron suggests, this complicated task may well take some time to

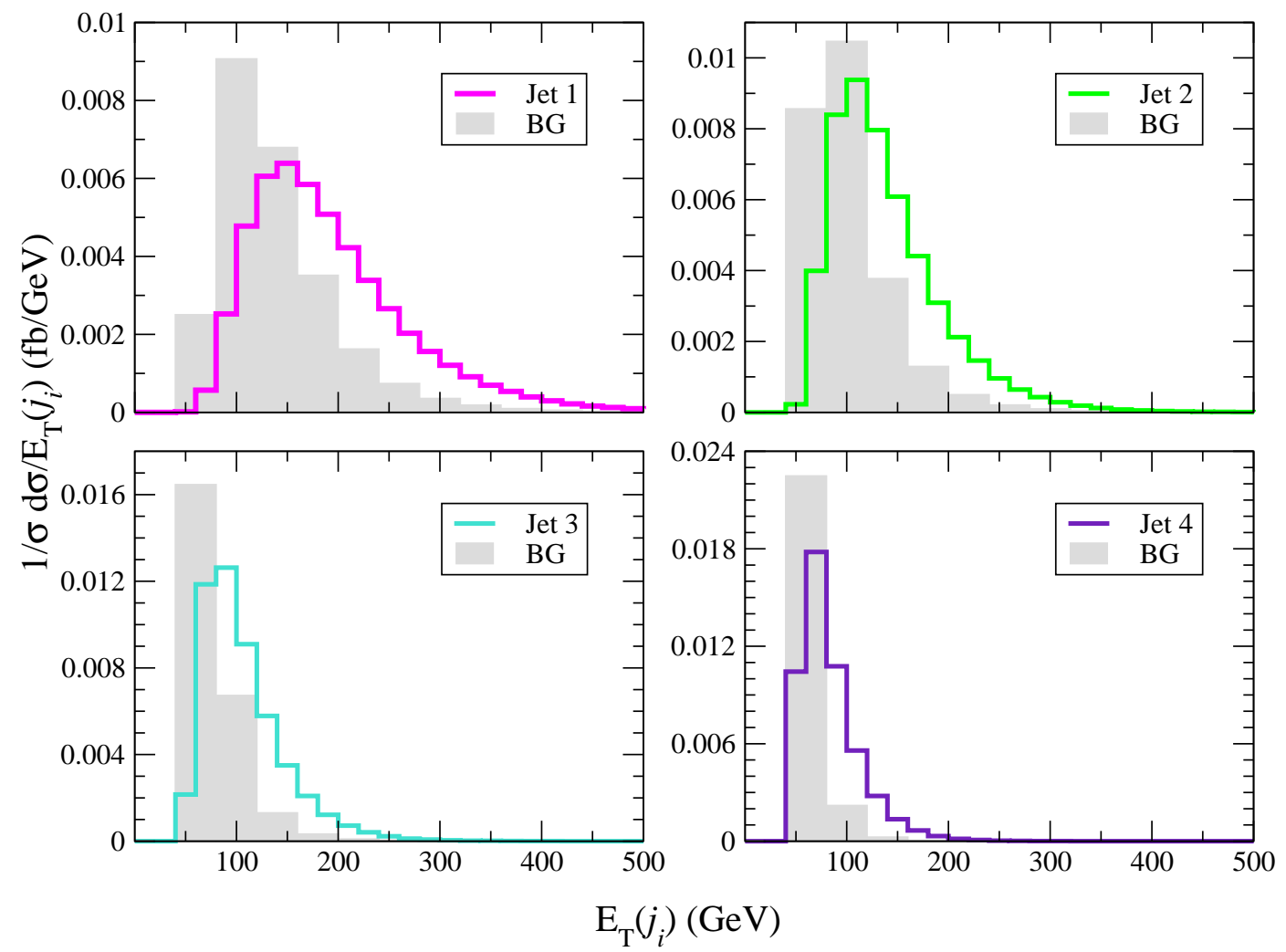

Figure 6: Plot of jet $E_{T}$ distributions for events with $\geq 4$ jets after requiring just $S_{T}>0.2$, from benchmark point $\mathrm{A}$; distributions for point $\mathrm{D}$ are nearly the same. 
complete. The same may also be true at the LHC, as many SM processes will have to be scrutinized first in order to properly calibrate the detector 34]. For this reason, SUSY searches using $E_{T}^{\text {miss }}$ as a crucial requirement may well take rather longer than a year to provide reliable results.

For this reason, Ref. [32] advocated to look for SUSY signal events by searching for a high multiplicity of detected objects, rather than inferred undetected objects, such as $E_{T}^{\text {miss }}$. In this vein, we show in Fig. 8 the jet multiplicity from SUSY signal (Pts. A and D) along with SM BG after cuts $C 1^{\prime}$, i.e with no $E_{T}^{\text {miss }}$ cut. We see that at low jet multiplicity, SM BG dominates the SUSY signal. However, signal/background increases with $n(j e t s)$ until at $n($ jets $) \sim 15$ finally signal overtakes $\mathrm{BG}$ in raw rate.

One can do better in detected $b$-jet multiplicity, $n_{b}$, as shown in Fig. 9. Since each gluino is expected to decay to two $b$-jets, we expect a high $n_{b}$ multiplicity in signal. In this case, BG dominates signal at low $n_{b}$, but signal overtakes BG around $n_{b} \simeq 4$.

The isolated lepton multiplicity $n_{\ell}$ is shown in Fig. 10 for signal and SM BG after cuts $C 1^{\prime}$. In this case, isolated leptons should be relatively common in gluino cascade decays. We see that signal exceeds BG already at $n_{\ell}=2$, and far exceeds BG at $n_{\ell}=3$ : at $n_{\ell}=3$, we have 35 (42) fb of signal cross section after cuts for point A (D) and no BG. In fact, high isolated lepton multiplicity was advocated in Ref. [32] in lieu of an $E_{T}^{\text {miss }}$ cut to search for SUSY with integrated luminosities of around $1 \mathrm{fb}^{-1}$ at LHC.

We also point out here that $\tilde{g} \tilde{g}$ production can lead to large rates for same-sign (SS) isolated dilepton production [35], while SM BG for this topology is expected to be small. We plot in Fig. 11 the rate of events from signal and SM BG for cuts $C 1^{\prime}$ plus a pair of isolated SS dileptons, versus jet multiplicity. While BG is large at low $n$ (jets), signal emerges from and dominates BG at higher jet multiplicities.

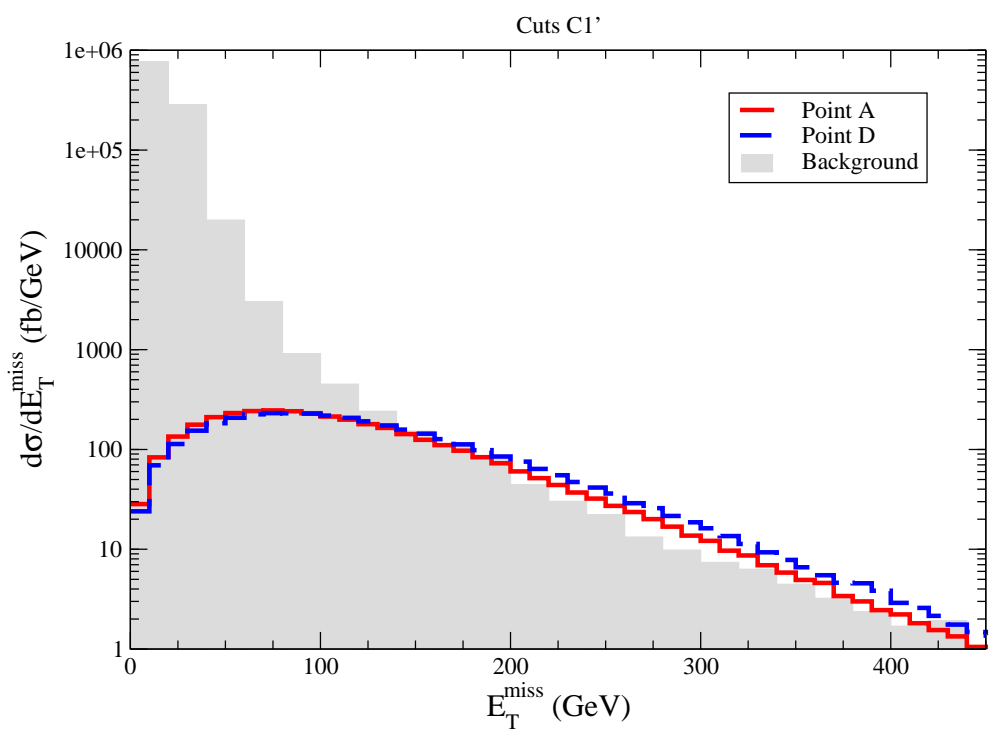

Figure 7: Plot of missing $E_{T}$ for events with $\geq 4$ jets after cuts $C 1^{\prime}$, from benchmark points A (full red line) and D (dashed blue line). 


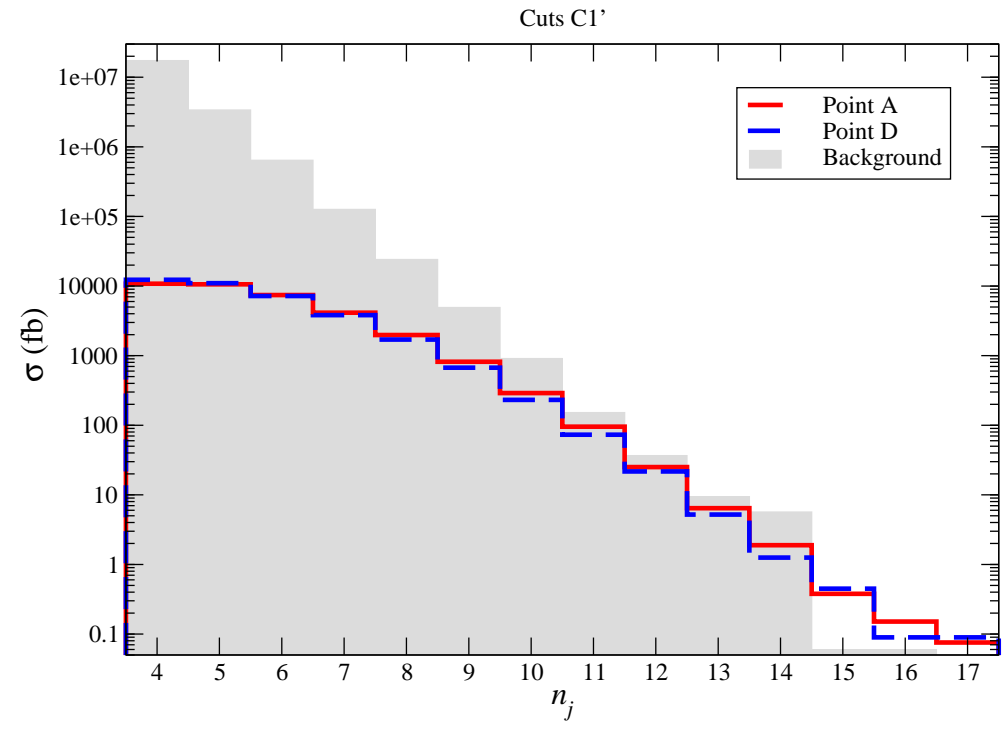

Figure 8: Plot of jet multiplicity from benchmark points A (full red line) and D (dashed blue line) after cuts $C 1^{\prime}$ along with SM backgrounds.

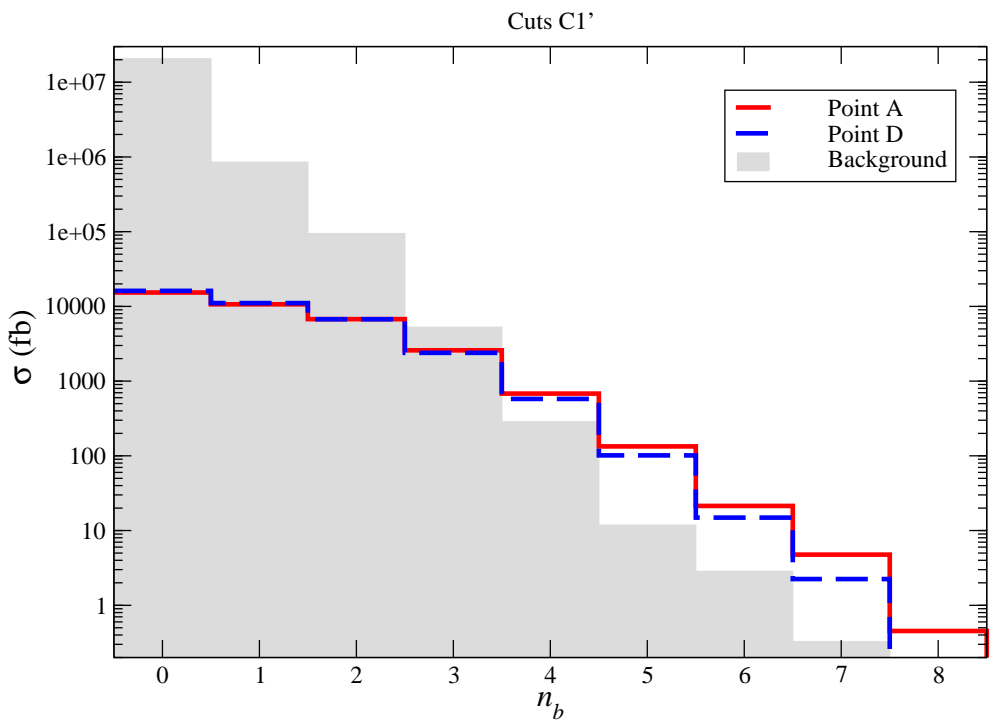

Figure 9: Plot of $b$-jet multiplicity from benchmark points A (full red line) and D (dashed blue line) after cuts $C 1^{\prime}$ along with SM backgrounds. 


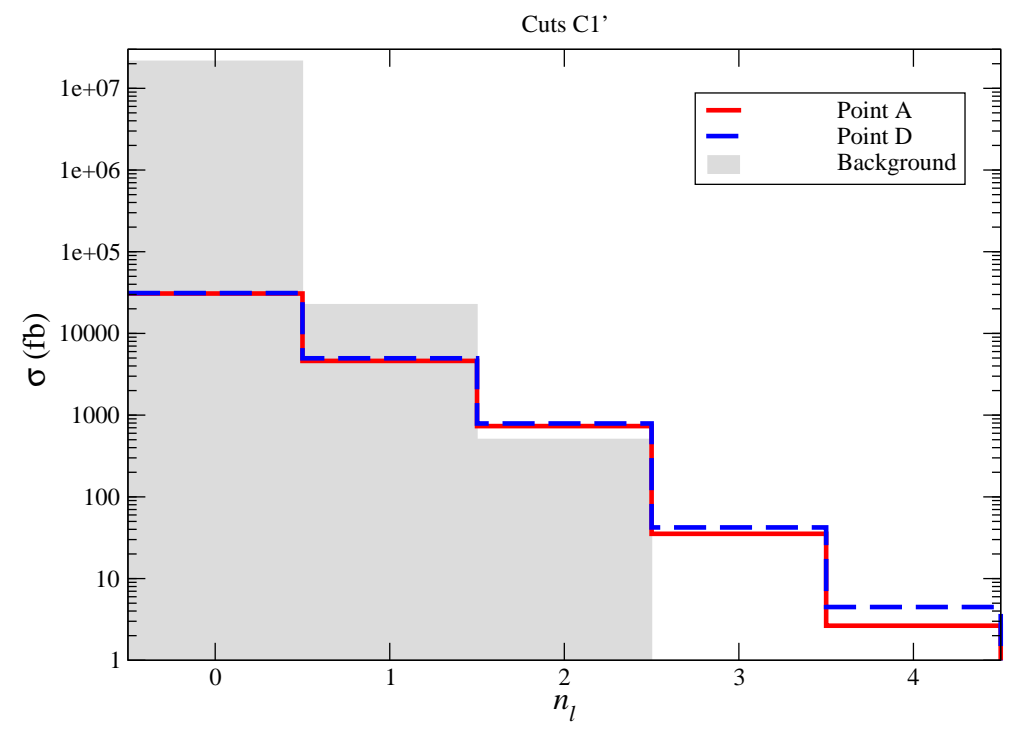

Figure 10: Plot of isolated lepton multiplicity from benchmark points A (full red line) and D (dashed blue line) after cuts $C 1^{\prime}$ along with SM backgrounds.

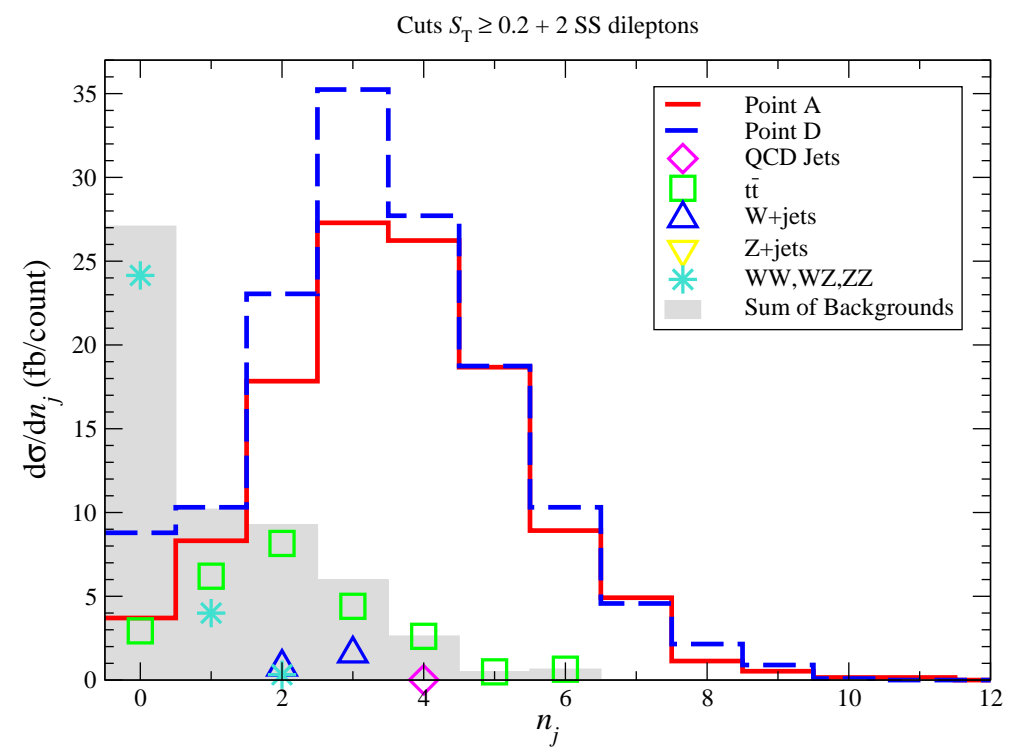

Figure 11: Plot of jet multiplicity in events with isolated SS dileptons from benchmark points A (full red line) and D (dashed blue line) after cut $S_{T}>0.2$ along with SM backgrounds. 


\section{Sparticle masses from gluino pair production}

There exists good prospects for sparticle mass measurements in Yukawa-unified SUSY models at the LHC. One reason is that sparticle pair production is dominated by a single reaction: gluino pair production. The other propitious circumstance is that the mass difference $m_{\tilde{\chi}_{2}^{0}}-m_{\tilde{\chi}_{1}^{0}}$ is highly favored to be bounded by $M_{Z}$. This means that $\tilde{\chi}_{2}^{0}$ decays dominantly into three body modes such as $\tilde{\chi}_{2}^{0} \rightarrow \tilde{\chi}_{1}^{0} \ell \bar{\ell}$ with a significant branching fraction, while the so-called "spoiler decay modes" $\tilde{\chi}_{2}^{0} \rightarrow \tilde{\chi}_{1}^{0} Z$ and $\tilde{\chi}_{2}^{0} \rightarrow \tilde{\chi}_{1}^{0} h$ are kinematically closed. The three body decay mode is important in that it yields a continuous distribution in $m(\ell \bar{\ell})$ which is bounded by $m_{\tilde{\chi}_{2}^{0}}-m_{\tilde{\chi}_{1}^{0}}$ : this kinematic edge can serve as the starting point for sparticle mass reconstruction in cascade decay events [36, 29].

As an example, we require cuts set $C 1^{\prime}$ plus the presence of a pair of same-flavor opposite-sign (SF/OS) isolated leptons. The resulting dilepton invariant mass distributions for points A and D are shown in Fig. 12. Furthermore, in Fig. 13, we plot the different-flavor subtracted distributions: $d \sigma / d m\left(\ell^{+} \ell^{-}\right)-d \sigma / d m\left(\ell^{+} \ell^{-\prime}\right)$, which allow for a subtraction of $e^{+} \mu^{-}$and $e^{-} \mu^{+}$pairs from processes like chargino pair production in cascade decay events. A clear peak at $m(\ell \bar{\ell})=M_{Z}$ is seen in the BG distribution. This comes mainly from QCD jet production events, since Isajet includes $W$ and $Z$ radiation in its parton shower algorithm (in the effective $W$ approximation). The signal displays a histogram easily visible above SM BG with a distinct cut-off at $m_{\tilde{\chi}_{2}^{0}}-m_{\tilde{\chi}_{1}^{0}}=73(52.5) \mathrm{GeV}$ for point A (D). Isajet contains the exact decay matrix elements in 3-body decay processes, and in these cases we see distributions that differ from pure phase space, and yield distributions skewed to higher $m(\ell \bar{\ell})$ values. This actually shows the influence of the virtual $Z$ in the decay diagrams, since the decay distribution is dominated by $Z^{*}$ exchange. The closer $m_{\tilde{\chi}_{2}^{0}}-m_{\tilde{\chi}_{1}^{0}}$ gets to $M_{Z}$, the more the $Z$-boson propagator pulls the dilepton mass distribution towards $M_{Z}$ [37]. The dilepton mass edge should be measureable to a precision of $\sim 50 \mathrm{MeV}$ according to Ref. 38.

For Yukawa-unified SUSY models, the branching fraction $B F\left(\tilde{g} \rightarrow b \bar{b} \tilde{\chi}_{2}^{0}\right)$ dominates at around $56 \%$. If one can identify events with a clean $\tilde{\chi}_{2}^{0} \rightarrow \ell \bar{\ell} \tilde{\chi}_{1}^{0}$ decay, then one might also try to extract the invariant mass of the associated two $b$-jets coming from the gluino decay, which should have a kinematic upper edge at $m_{\tilde{g}}-m_{\tilde{\chi}_{2}^{0}} \simeq 267$ (283) GeV for point A (D). A second, less pronounced endpoint is expected at $m_{\tilde{g}}-m_{\tilde{\chi}_{1}^{0}} \simeq 340$ (335) GeV due to $\tilde{g} \rightarrow b \bar{b} \tilde{\chi}_{1}^{0}$ decays which have $\sim 16 \%$ branching ratio. A third endpoint can also occur from $\tilde{\chi}_{2}^{0} \rightarrow \tilde{\chi}_{1}^{0} b \bar{b}$ decay where $m_{\tilde{\chi}_{2}^{0}}-m_{\tilde{\chi}_{1}^{0}}=73(52.5) \mathrm{GeV}$, respectively.

The high multiplicity of $b$-jets (typically two from each gluino decay) however poses a serious combinatorics problem in extracting the $b \bar{b}$ invariant-mass distribution. In a first attempt, we required at least two tagged $b$-jets along with cuts $C 1^{\prime}$ and $\mathrm{SF} / \mathrm{OS}$ dileptons, and plotted the minimum invariant mass of all the $b$-jets in the event. The resulting distributions for points $\mathrm{A}$ and $\mathrm{D}$ peaked around $m(b \bar{b}) \sim 100 \mathrm{GeV}$, with a distribution tail extending well beyond the above-mentioned kinematic endpoints. It was clear that we were frequently picking up wrong $b$-jet pairs, with either each $b$ originating from a different gluino, or one or both $b$ 's originating from a $\tilde{\chi}_{2}^{0} \rightarrow \tilde{\chi}_{1}^{0} b \bar{b}$ decay. A more sophisticated procedure to pair the correct $b$-jets is required for the $b \bar{b}$ invariant-mass distribution. 


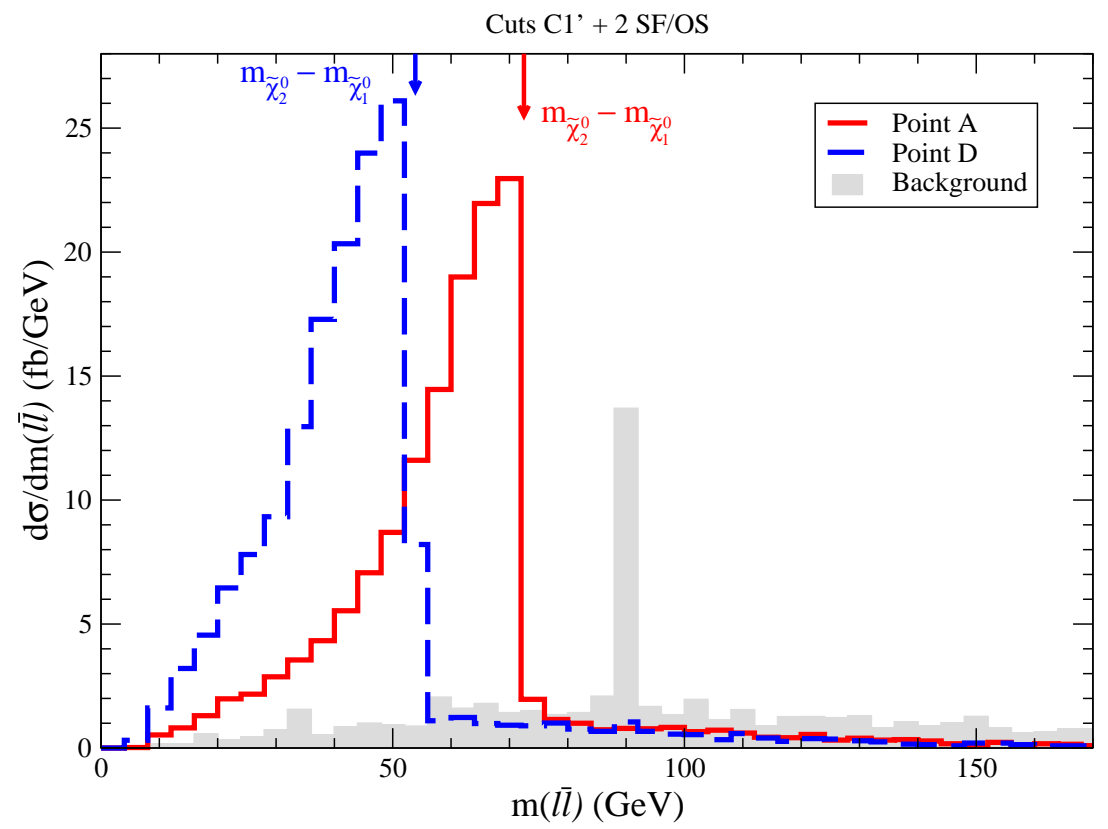

Figure 12: SF/OS dilepton invariant mass distribution after cuts $C 1^{\prime}$ from benchmark points A (full red line) and D (dashed blue line) along with SM backgrounds.

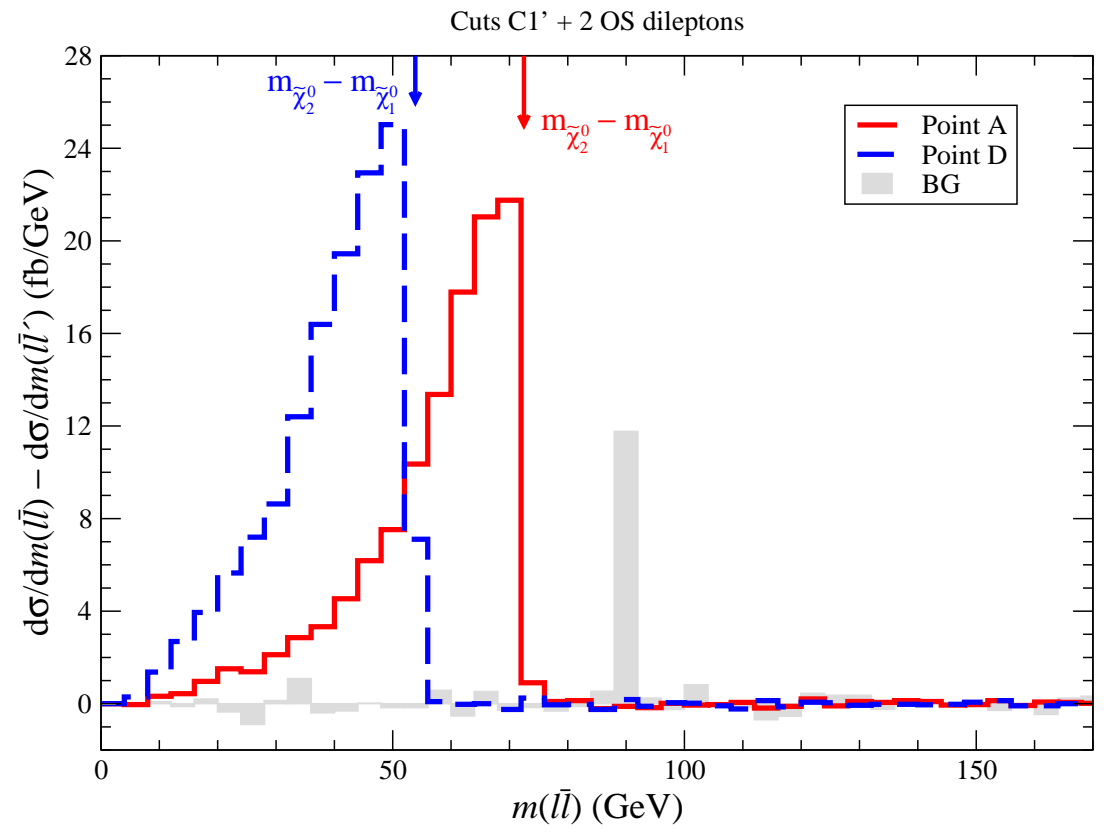

Figure 13: Same as Fig. 12 but for same-flavor minus different-flavor subtracted invariant-mass. 


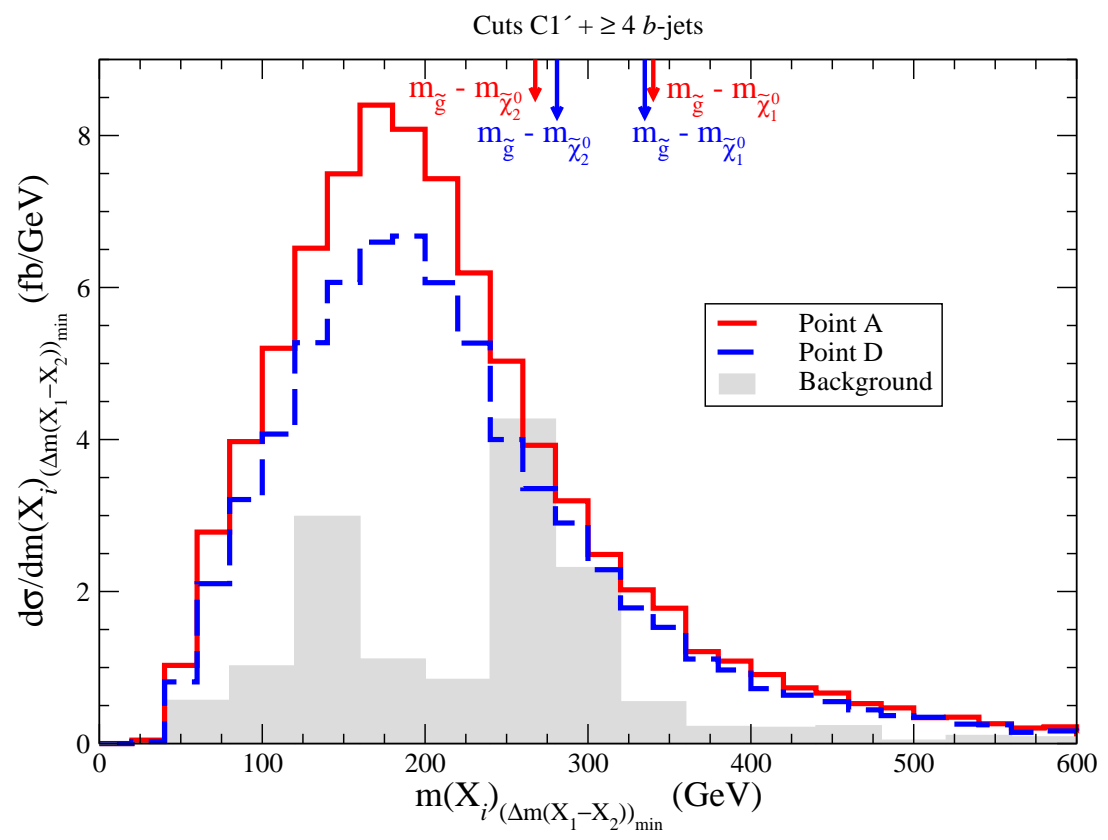

Figure 14: Plot of $m\left(X_{1,2}\right)$ from benchmark points A and D along with SM backgrounds in events with cuts $C 1^{\prime}$ plus $\geq 4 b$-jets and minimizing $\Delta m\left(X_{1}-X_{2}\right)$; see text for details.

Parton-level Monte Carlo simulations revealed that the two hardest (highest $E_{T}$ ) $b$ jets almost always originated from different gluinos. Thus, we require events with at least four tagged $b$-jets (along with cuts $C 1^{\prime}$ ) and combine the hardest $b$-jet with either the 3rd or 4 th hardest $b$-jet, creating an object $X_{1}(b b)$. Moreover, we combine the 2 nd hardest $b$-jet with the 4 th or 3 rd hardest $b$-jet, creating an object $X_{2}(b b)$. Next, we calculate $\Delta m\left(X_{1}-X_{2}\right) \equiv\left|m\left(X_{1}\right)-m\left(X_{2}\right)\right| /\left(m\left(X_{1}\right)+m\left(X_{2}\right)\right)$. We select the set of $b b$ clusters which has the minimum value of $\Delta m\left(X_{1}-X_{2}\right)$, and plot the invariant mass of both the clusters. This procedure produces a sharp kinematic edge in $m(b b)$ in parton level simulations. ${ }^{4}$ The resulting distribution from Isajet is shown in Fig. 14 for points A and D. The distribution peaks at a higher value of $m\left(X_{i}\right)(i=1,2)$, and is largely bounded by the kinematic endpoints, although a tail still extends to high $m\left(X_{i}\right)$. Part of the high $m\left(X_{i}\right)$ tail is due to the presence of $\tilde{g} \rightarrow \tilde{\chi}_{1}^{0} b \bar{b}$ decays, which have a higher kinematic endpoint than the $\tilde{g} \rightarrow \tilde{\chi}_{2}^{0} b \bar{b}$ decays, and of $\tilde{\chi}_{2}^{0} \rightarrow \tilde{\chi}_{1}^{0} b \bar{b}$ decays. In addition, there is a non-negligible background contribution, indicated by the gray histogram.

We can do much better, albeit with reduced statistics, by requiring in addition the presence of a pair of SF/OS dileptons. Applying the same procedure as described above, we arrive at the distribution shown in Fig. 15. In this case, the SM BG is greatly reduced, and two mass edges begin to appear.

It should also be possible to combine the invariant mass of the SF/OS dilepton pair

\footnotetext{
${ }^{4}$ We have also tried other methods such as picking the $X_{1}-X_{2}$ pair with maximum $\Delta \phi\left(X_{1}-X_{2}\right)$, maximum $\Delta R\left(X_{1}-X_{2}\right)$, minimum $\delta p_{T}\left(X_{1}-X_{2}\right)$ and minimum average invariant mass $\operatorname{avg}\left(m\left(X_{1}\right), m\left(X_{2}\right)\right)$. We also tried to separate the resulting $b$ jets into hemispheres. In the end, the best amount of correct assignment was achieved with the choice of the $\Delta m\left(X_{1}-X_{2}\right)_{\min }$.
} 


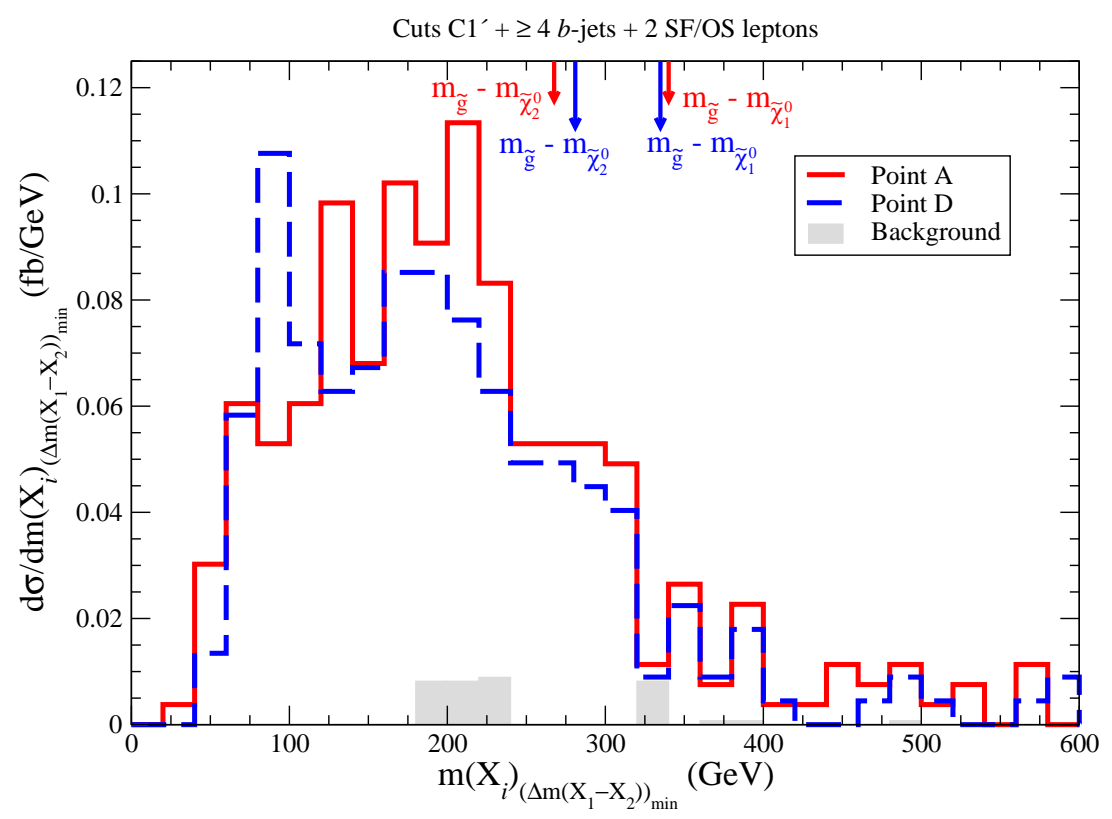

Figure 15: Same as Fig. 14 but requiring in addition a pair of SF/OS leptons.

with a $b \bar{b}$ pair. Requiring cuts $C 1^{\prime}$ plus $\geq 2 b$-jets plus a pair of SF/OS dileptons (with $\left.m(\ell \bar{\ell})<m_{\tilde{\chi}_{2}^{0}}-m_{\tilde{\chi}_{1}^{0}}\right)$, we reconstruct $m(b b \ell \bar{\ell})$. The result is shown in Fig. 16. While the distribution peaks at $m(\ell \bar{\ell} b \bar{b}) \sim 300 \mathrm{GeV}$, a kinematic edge at $m_{\tilde{g}}-m_{\tilde{\chi}_{1}^{0}} \sim 340 \mathrm{GeV}$ is also visible (along with a mis-identification tail extending to higher invarant masses).

We try to do better, again with a loss of statistics, by requiring $\geq 4 b$-jets instead of $\geq 2$, and combining the $b b$ clusters into objects $X_{1}$ and $X_{2}$ as described above. We again choose the set of clusters which give the minimum of $\Delta m\left(X_{1}-X_{2}\right)$ and combine each of these clusters with the $\ell \bar{\ell}$ pair. We take the minimum of the two $m\left(X_{i} \ell \bar{\ell}\right)$ values, and plot the distribution in Fig. 17. In this case, the SM BG is even more reduced, and the $m_{\tilde{g}}-m_{\tilde{\chi}_{1}^{0}}$ mass edge seems somewhat more apparent.

According to [38], measurements of hadronic mass edges can be made with a precision of roughly $10 \%$. Nevertheless, from the kinematic distributions discussed above we can only determine mass differences. There is still not enough information to extract absolute masses, i.e. each of $m_{\tilde{g}}, m_{\tilde{\chi}_{2}^{0}}$ and $m_{\tilde{\chi}_{1}^{0}}$. However, it is pointed out in Ref. [39] that in cases (such as the focus point region of minimal supergravity) where sparticle pair production occurs nearly purely from $\tilde{g} \tilde{g}$ production, and when the dominant $\tilde{g}$ branching fractions are known (from a combination of theory and experiment), then the total $\tilde{g} \tilde{g}$ production cross section after cuts allows for an absolute measurement of $m_{\tilde{g}}$ to about an $8 \%$ accuracy. These conditions should apply to our Yukawa-unified SUSY cases, if we assume the $\sim 56 \%$ branching fraction for $\tilde{g} \rightarrow b \bar{b} \tilde{\chi}_{2}^{0}$ decay (from theory). The study of Ref. [39] required that one fulfill the cuts $C 2$ which gave robust gluino pair production signal along with small SM backgrounds: 


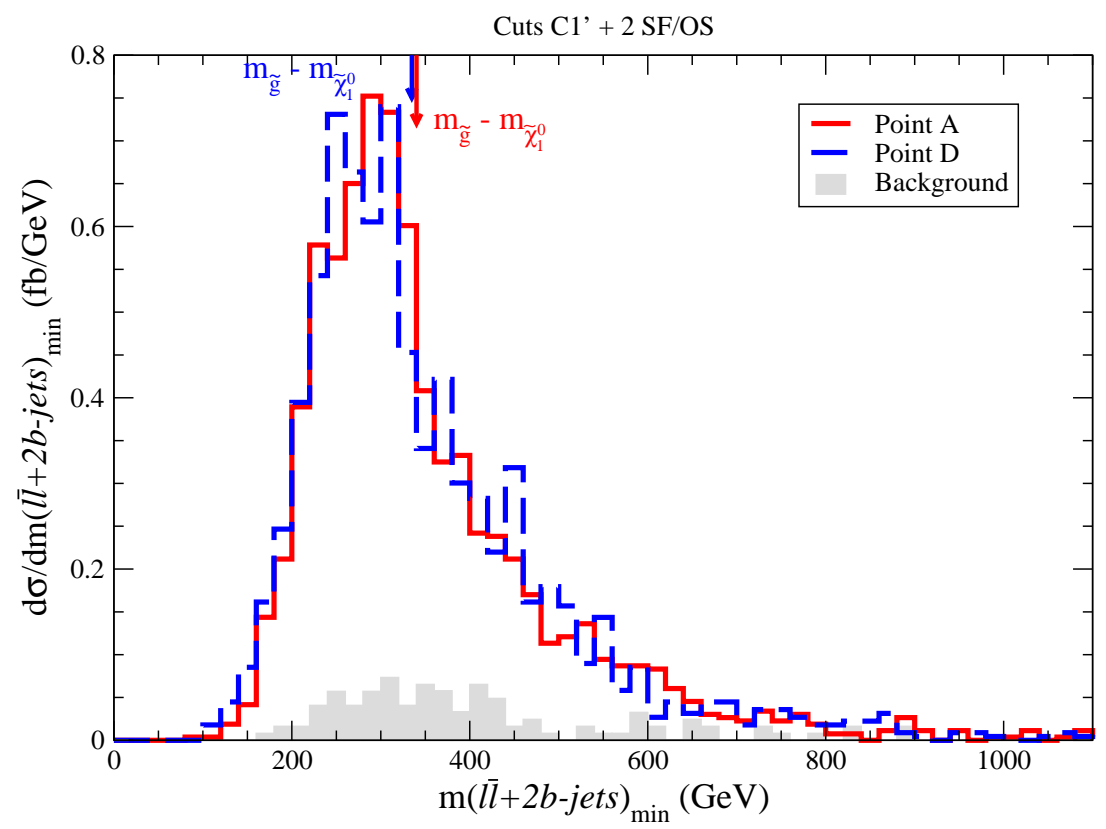

Figure 16: Plot of $m\left(b b \ell^{+} \ell^{-}\right)_{\min }$ from points A and D along with SM backgrounds.

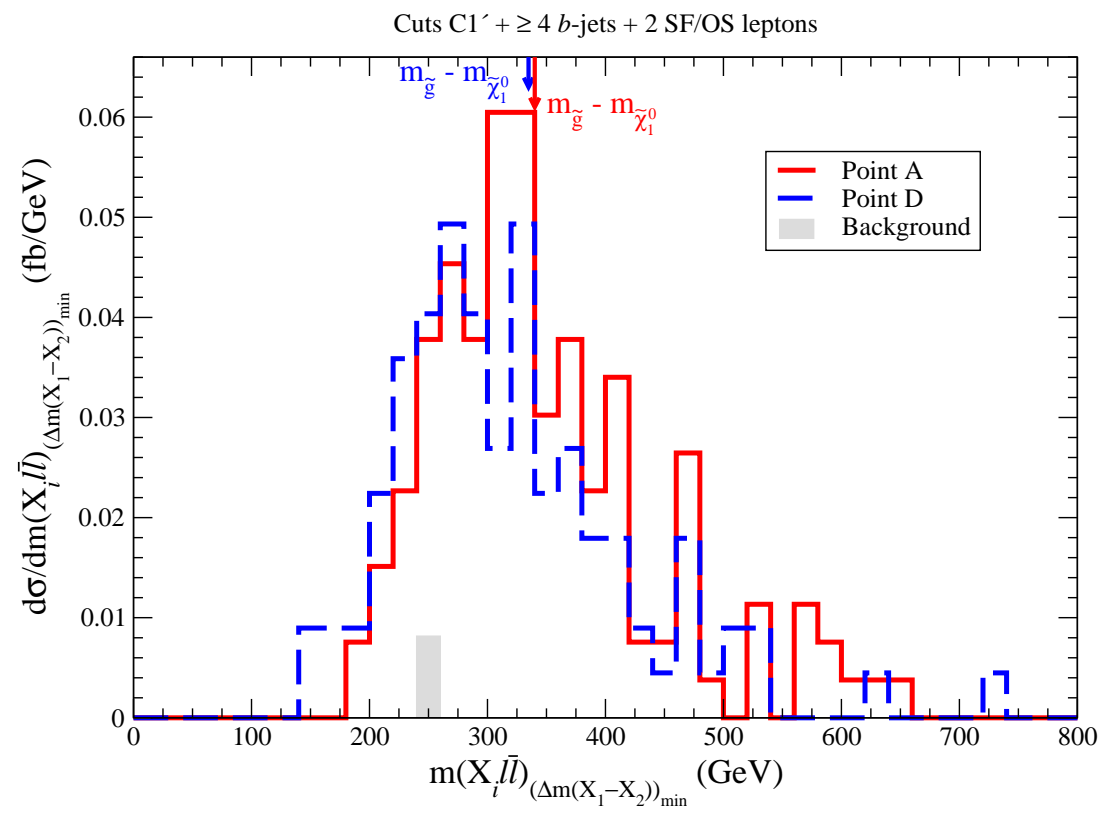

Figure 17: Plot of $m\left(X_{1,2} \ell^{+} \ell^{-}\right)_{\min }$ from points $\mathrm{A}$ and D, minimizing $\Delta m\left(X_{1}-X_{2}\right)$ as explained in the text, along with SM backgrounds. 


\section{C2 cuts:}

$$
\begin{array}{r}
E_{T}^{\text {miss }}>\left(\max \left(100 \mathrm{GeV}, 0.2 M_{\text {eff }}\right),\right. \\
n(\text { jets }) \geq 7, \\
n(b-j e t s) \geq 2, \\
E_{T}(j 1, j 2-j 7)>100,50 \mathrm{GeV}, \\
A_{T}>1400 \mathrm{GeV}, \\
S_{T} \geq 0.2,
\end{array}
$$

where $A_{T}$ is the augmented effective mass $A_{T}=E_{T}^{\text {miss }}+\sum_{\text {leptons }} E_{T}+\sum_{\text {jets }} E_{T}$. In this case, the summed SM background was about $1.6 \mathrm{fb}$, while signal rate for Point A (D) is $57.3(66.2) \mathrm{fb}$. The total cross section after cuts varies strongly with $m_{\tilde{g}}$, allowing an extraction of $m_{\tilde{g}}$ to about $8 \%$ for $100 \mathrm{fb}^{-1}$ integrated luminosity, after factoring in QCD and branching fraction uncertainties in the total rate. Once an absolute value of $m_{\tilde{g}}$ is known, then $m_{\tilde{\chi}_{2}^{0}}$ and $m_{\tilde{\chi}_{1}^{0}}$ can be extracted to about $10 \%$ accuracy from the invariant mass edge information.

\section{Trilepton signal from $\tilde{\chi}_{1} \tilde{\chi}_{2}^{0}$ production}

While the signal from gluino pair production at the LHC from Yukawa-unified SUSY models will be very robust, it will be useful to have a confirming SUSY signal in an alternative channel. From Fig. 3, we see that there also exists substantial cross sections for $\tilde{\chi}_{1}^{ \pm} \tilde{\chi}_{1}^{0}$, $\tilde{\chi}_{1}^{+} \tilde{\chi}_{1}^{-}$and $\tilde{\chi}_{1}^{ \pm} \tilde{\chi}_{2}^{0}$ production. The $\tilde{\chi}_{1}^{ \pm} \rightarrow \tilde{\chi}_{1}^{0} f \bar{f}^{\prime}$ and $\tilde{\chi}_{2}^{0} \rightarrow \tilde{\chi}_{1}^{0} f \bar{f}$ decays (here $f$ stands for any of the SM fermions) are dominated by $W$ and $Z$ exchange, respectively, so that in this case the branching fractions $B F\left(\tilde{\chi}_{1}^{ \pm} \rightarrow \tilde{\chi}_{1}^{0} f \bar{f}^{\prime}\right)$ are similar to $B F\left(W^{ \pm} \rightarrow f \bar{f}^{\prime}\right)$ and $B F\left(\tilde{\chi}_{2}^{0} \rightarrow \tilde{\chi}_{1}^{0} f \bar{f}\right)$ is similar to $Z \rightarrow f \bar{f}$.

The $\tilde{\chi}_{1}^{ \pm} \tilde{\chi}_{1}^{0} \rightarrow \tilde{\chi}_{1}^{0} q \bar{q}^{\prime}+\tilde{\chi}_{1}^{0}$ process will be difficult to observe at LHC since the final state jets and $E_{T}^{\text {miss }}$ will be relatively soft, and likely buried under SM background. Likewise, the $\tilde{\chi}_{1}^{-} \tilde{\chi}_{1}^{0} \rightarrow \tilde{\chi}_{1}^{0} \ell \bar{\nu}_{\ell} \tilde{\chi}_{1}^{0}$ signal will be buried under a huge BG from $W \rightarrow \ell \bar{\nu}_{\ell}$ production. The $\tilde{\chi}_{1}^{+} \tilde{\chi}_{1}^{-}$production reaction will also be difficult to see at LHC. The purely hadronic final state will likely be buried under QCD and $Z+$ jets $\mathrm{BG}$, while the lepton plus jets final state will be buried under $W+$ jets $\mathrm{BG}$. The dilepton final state will be difficult to extract from $W^{+} W^{-}$and $t \bar{t}$ production.

The remaining reaction, $\tilde{\chi}_{1}^{ \pm} \tilde{\chi}_{2}^{0}$ production, yields a trilepton final state from $\tilde{\chi}_{1}^{ \pm} \rightarrow$ $\tilde{\chi}_{1}^{0} \ell \bar{\nu}_{\ell}$ and $\tilde{\chi}_{2}^{0} \rightarrow \tilde{\chi}_{1}^{0} \ell \bar{\ell}$ decays which in many cases is observable above SM BG. The LHC reach for $\tilde{\chi}_{1}^{ \pm} \tilde{\chi}_{2}^{0} \rightarrow 3 \ell+E_{T}^{\text {miss }}$ production was mapped out in Ref. [40], and the reach was extended into the hyperbolic branch/focus point (HB/FP) region in Ref. 41]. The method was to use the cut set SC2 from Ref. [37] but as applied to the LHC. For the clean trilepton signal from $\tilde{\chi}_{1}^{ \pm} \tilde{\chi}_{2}^{0} \rightarrow 3 \ell+E_{T}^{\text {miss }}$ production, we require:

- three isolated leptons with $p_{T}(\ell)>20 \mathrm{GeV}$ and $\left|\eta_{\ell}\right|<2.5$,

- SF/OS dilepton mass $20 \mathrm{GeV}<m\left(\ell^{+} \ell^{-}\right)<81 \mathrm{GeV}$, to avoid BG from photon and $Z$ poles in the $2 \rightarrow 4$ process $q \bar{q}^{\prime} \rightarrow \ell \bar{\ell} \ell^{\prime} \bar{\nu}_{\ell}$, 


\begin{tabular}{lrccc}
\hline \multicolumn{1}{l}{ process } & events & $\sigma(\mathrm{fb})$ & after cuts $(\mathrm{fb})$ \\
\hline$t \bar{t}$ & & $3 \times 10^{6}$ & $4.9 \times 10^{5}$ & - \\
$W W, Z Z, W Z$ & & $5 \times 10^{5}$ & $8.0 \times 10^{4}$ & - \\
$W^{*} Z^{*}, W^{*} \gamma^{*} \rightarrow \ell \bar{\ell} \ell^{\prime} \nu_{\ell^{\prime}}$ & & $10^{6}$ & - & 0.7 \\
\hline Total BG & - & $10^{6}$ & $7.6 \times 10^{4}$ & 3.4 \\
\hline Point A: & $S / B \rightarrow$ & - & - & 4.86 \\
& $S / \sqrt{S+B}\left(10 \mathrm{fb}^{-1}\right) \rightarrow$ & - & - & 5.31 \\
Point D: & - & $10^{6}$ & $9.0 \times 10^{4}$ & 4.1 \\
& $S / B \rightarrow$ & - & - & 5.86 \\
& $S / \sqrt{S+B}\left(10 \mathrm{fb}^{-1}\right) \rightarrow$ & - & - & 5.92 \\
\hline
\end{tabular}

Table 3: Clean trilepton signal after cuts listed in the text.

- a transverse mass veto $60 \mathrm{GeV}<M_{T}\left(\ell, E_{T}^{\text {miss }}\right)<85 \mathrm{GeV}$ to reject on-shell $W$ contributions,

- $E_{T}^{\mathrm{miss}}>25 \mathrm{GeV}$ and,

- veto events with $n($ jets $) \geq 1$.

The resulting BG levels and signal rates for points A and D are listed in Table 3 . The $2 \rightarrow 2$ processes are calculated with Isajet, while the $2 \rightarrow 4$ processes are calculated at parton level using Madgraph1 42]. The combination of hard lepton $p_{T}$ cuts and the requirement that $n$ (jets) $=0$ leaves us with no $2 \rightarrow 2$ background, while the parton level $2 \rightarrow 4$ BG remains at $0.7 \mathrm{fb}$. Here, we see that signal from the two Yukawa-unified points well exceeds background.

In the clean $3 \ell$ channel, since two of the leptons ought to come from $\tilde{\chi}_{2}^{0} \rightarrow \tilde{\chi}_{1}^{0} \ell \bar{\ell}$ decay, they should display a confirmatory dilepton mass edge at $m_{\tilde{\chi}_{2}^{0}}-m_{\tilde{\chi}_{1}^{0}}$ as is evident in the gluino pair production events, where the dileptons are accompanied by high jet multiplicity. The distribution in $m\left(\ell^{+} \ell^{-}\right)$is shown in Fig. 18. Event rates are seen to be lower than those from $\tilde{g} \tilde{g}$ production. Integrated luminosity needed for a discovery with $5 \sigma$ significance would be $2.83 \mathrm{fb}^{-1}$ for pt. A and $1.5 \mathrm{fb}^{-1}$ for pt. $\mathrm{D}^{5}$.

\section{Summary and conclusions}

Simple SUSY grand unified models based on the gauge group $S O(10)$ may have $t-b-\tau$ Yukawa coupling unification in addition to gauge group and matter unification. By assuming the MSSM is the effective field theory valid below $M_{G U T}$, we can, starting with weak scale fermion masses as boundary conditions, check whether or not these third generation Yukawa couplings actually unify. The calculation depends sensitively on the entire SUSY particle mass spectrum, mainly through radiative corrections to the $b, t$ and $\tau$ masses. It

\footnotetext{
${ }^{5}$ Significance is defined as $S / \sqrt{(S+B)}$
} 


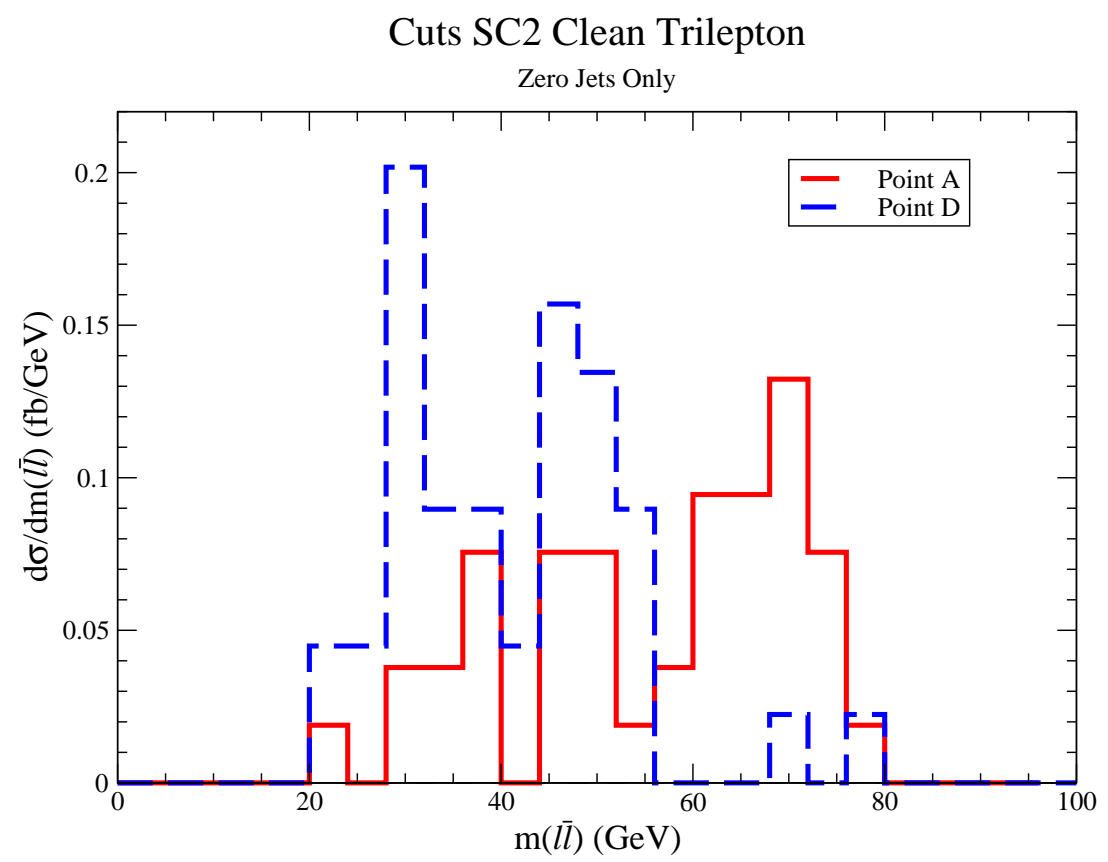

Figure 18: Plot of $m\left(\ell^{+} \ell^{-}\right)$in the clean trilepton channel from points A and D along with SM backgrounds.

was found in previous works that $t-b-\tau$ Yukawa coupling unification can occur, but only for very restrictive soft SUSY breaking parameter boundary conditions valid at the GUT scale, leading to a radiatively induced inverted mass hierarchy amongst the sfermion masses. While squarks and sleptons are expected to be quite heavy, gluinos, winos and binos are expected to be quite light, and will be produced at large rates at the CERN LHC.

We expect LHC collider events from Yukawa-unified SUSY models to be dominated by gluino pair production at rates of $(30-150) \times 10^{3} \mathrm{fb}$. The $\tilde{g}$ s decay via 3 -body modes into $b \bar{b} \tilde{\chi}_{2}^{0}, b \bar{b} \tilde{\chi}_{1}^{0}$ and $t b \tilde{\chi}_{1}^{ \pm}$, followed by leptonic or hadronic 3 -body decays of the $\tilde{\chi}_{2}^{0}$ and $\tilde{\chi}_{1}^{ \pm}$. A detailed simulation of signal and SM BG processes shows that signal should be easily visible above $\mathrm{SM} \mathrm{BG}$ in the $\geq 4$ jets plus $\geq 3 \ell$ channel, even without using the $E_{T}^{\text {miss }}$ variable, with about $1 \mathrm{fb}^{-1}$ of integrated luminosity.

If Yukawa-unified signals from $\tilde{g} \tilde{g}$ production are present, then at higher integrated luminosities, mass edges in the $m\left(\ell^{+} \ell^{-}\right), m(b \bar{b})$ and $m\left(b \bar{b} \ell^{+} \ell^{-}\right)$channels along with total cross section rates (which depend sensitively on the value of $m_{\tilde{g}}$ ) should allow for sparticle mass reconstruction of $m_{\tilde{g}}, m_{\tilde{\chi}_{2}^{0}}$ and $m_{\tilde{\chi}_{1}^{0}}$ to $\mathcal{O}(10 \%)$ accuracy for $\sim 100 \mathrm{fb}^{-1}$ of integrated luminosity. The gluino pair production signal can be corroborated by another signal in the clean trilepton channel from $\tilde{\chi}_{1} \tilde{\chi}_{2}^{0} \rightarrow 3 \ell+E_{T}^{\text {miss }}$, which should also be visible at higher integrated luminosities. Thus, based on the study presented here, we expect LHC to either discover or rule out $t-b-\tau$ Yukawa-unified SUSY models within the first year or two of operation. 


\section{Acknowledgments}

This research was supported in part by the U.S. Department of Energy grant numbers DE-FG02-97ER41022. This work is also part of the French ANR project ToolsDMColl, BLAN07-2-194882. SS acknowledges financial support by Turkish Atomic Energy Authority. SK thanks the CERN Theory unit for hospitality during stages of this work.

\section{References}

[1] H. Georgi, in Proceedings of the American Institue of Physics, edited by C. Carlson (1974); H. Fritzsch and P. Minkowski, Ann. Phys. 93, 193 (1975); M. Gell-Mann, P. Ramond and R. Slansky, Rev. Mod. Phys. 50, 721 (1978). For recent reviews, see R. Mohapatra, hep-ph/9911272 (1999) and S. Raby, in Rept. Prog. Phys. 67 (2004) 755. For additional perspective, see G. Altarelli and F. Feruglio, hep-ph/0405048.

[2] M. Gell-Mann, P. Ramond and R. Slansky, in Supergravity, Proceedings of the Workshop, Stony Brook, NY 1979 (North-Holland, Amsterdam); T. Yanagida, KEK Report No. 79-18, 1979; R. Mohapatra and G. Senjanovic, Phys. Rev. Lett. 44 (1980) 912.

[3] Y. Kawamura, Prog. Theor. Phys. 105 (2001) 999; G. Altarelli and F. Feruglio, Phys. Lett. B 511 (2001) 257; L. Hall and Y. Nomura, Phys. Rev. D 64 (2001) 055003; A. Hebecker and J. March-Russell, Nucl. Phys. B 613 (2001) 3; A. Kobakhidze, Phys. Lett. B 514 (2001) 131.

[4] D. Auto, H. Baer, C. Balazs, A. Belyaev, J. Ferrandis and X. Tata, J. High Energy Phys. 0306 (2003) 023 .

[5] T. Blazek, R. Dermisek and S. Raby, Phys. Rev. D 65 (2002) 115004.

[6] The Tevatron Electroweak Working group (CDF and D0 Collaborations), arXiv:0803.1683.

[7] H. Baer and J. Ferrandis, Phys. Rev. Lett. 87 (2001) 211803.

[8] H. Baer, S. Kraml, S. Sekmen and H. Summy, J. High Energy Phys. 0803 (2008) 056.

[9] T. Blazek, R. Dermisek and S. Raby, Phys. Rev. Lett. 88 (2002) 111804.

[10] R. Dermisek, S. Raby, L. Roszkowski and R. Ruiz de Austri, J. High Energy Phys. 0304 (2003) 037 and J. High Energy Phys. 0509 (2005) 029; M. Albrecht, W. Altmannshofer, A. Buras, D. Guadagnoli and D. Straub, J. High Energy Phys. 0710 (2007) 055; W. Altmannshofer, D. Guadagnoli, S. Raby and D. Straub, arXiv:0801.4363 (2008).

[11] J. Feng, C. Kolda and N. Polonsky, Nucl. Phys. B 546 (1999) 3; J. Bagger, J. Feng and N. Polonsky, Nucl. Phys. B 563 (1999) 3; J. Bagger, J. Feng, N. Polonsky and R. Zhang, Phys. Lett. B 473 (2000) 264; H. Baer,P. Mercadante and X. Tata, Phys. Lett. B 475 (2000) 289; H. Baer, C. Balazs, M. Brhlik, P. Mercadante, X. Tata and Y. Wang, Phys. Rev. D 64 (2001) 015002; see also H. Baer, M. Diaz, P. Quintana and X. Tata, J. High Energy Phys. 0004 (2000) 016.

[12] F. Paige, S. Protopopescu, H. Baer and X. Tata, hep-ph/0312045; http://www.hep.fsu.edu/ isajet/

[13] H. Baer, J. Ferrandis, S. Kraml and W. Porod, Phys. Rev. D 73 (2006) 015010.

[14] S. P. Martin and M. Vaughn, Phys. Rev. D 50 (1994) 2282.

[15] H. E. Haber, R. Hempfling and A. Hoang, Z. Physik C 75 (1996) 539. 
[16] R. Hempfling, Phys. Rev. D 49 (1994) 6168; L. J. Hall, R. Rattazzi and U. Sarid, Phys. Rev. D 50 (1994) 7048; M. Carena et al., Nucl. Phys. B 426 (1994) 269.

[17] D. Pierce, J. Bagger, K. Matchev and R. Zhang, Nucl. Phys. B 491 (1997) 3.

[18] D. Auto, H. Baer and A. Belyaev and T. Krupovnickas, J. High Energy Phys. 0410 (2004) 066 .

[19] D. N. Spergel et al. (WMAP Collaboration), Astrophys. J. Supp., 170 (2007) 377.

[20] L. Covi, J. E. Kim and L. Roszkowski, Phys. Rev. Lett. 82 (1999) 4180; L. Covi, H. B. Kim, J. E. Kim and L. Roszkowski, J. High Energy Phys. 0105 (2001) 033.

[21] E. J. Chun, J. E. Kim and H. P. Nilles, Phys. Lett. B 287 (1992) 123.

[22] K. Jedamzik, M. LeMoine and G. Moultaka, JCAP0607 (2006) 010.

[23] A. Brandenburg and F. Steffen, JCAP0408 (2004) 008.

[24] K. Kohri, T. Moroi and A. Yotsuyanagi, Phys. Rev. D 73 (2006) 123511.

[25] W. Buchmuller, P. Di Bari and M. Plumacher, Annal. Phys. 315 (2005) 305.

[26] G. Lazarides and Q. Shafi, Phys. Lett. B 258 (1991) 305; K. Kumekawa, T. Moroi and T. Yanagida, Prog. Theor. Phys. 92 (1994) 437; T. Asaka, K. Hamaguchi, M. Kawasaki and T. Yanagida, Phys. Lett. B 464 (1999) 12.

[27] H. Baer and H. Summy, Phys. Lett. B 666 (2008) 5.

[28] H. Baer, J. Ellis, G. Gelmini, D. Nanopoulos and X. Tata, Phys. Lett. B 161 (1985) 175; G. Gamberini, Z. Physik C 30 (1986) 605; H. Baer, V. Barger, D. Karatas and X. Tata, Phys. Rev. D 36 (1987) 96; R. M. Barnett, J. F. Gunion and H. Haber, Phys. Rev. D 37 (1988) 1892; H. Baer, X. Tata and J. Woodside, Phys. Rev. D 42 (1990) 1568; A. Bartl, W. Majerotto, B. Mösslacher, N. Oshimo and S. Stippel, Phys. Rev. D 43 (1991) 2214 H. Baer, M. Bisset, X. Tata and J. Woodside, Phys. Rev. D 46 (1992) 303; A. Bartl, W. Majerotto and W. Porod, Z. Physik C 64 (1994) 499; A. Djouadi, Y. Mambrini and M. Mühlleitner, Eur. Phys. J. C 20 (2001) 563; J. Hisano, K. Kawagoe and M. Nojiri, Phys. Rev. D 68 (2003) 035007.

[29] H. Baer, C. H. Chen, F. Paige and X. Tata, Phys. Rev. D 50 (1994) 4508.

[30] W. Beenakker, R. Hopker and M. Spira, hep-ph/9611232 (1996).

[31] P. G. Mercadante, J. K. Mizukoshi and X. Tata, Phys. Rev. D 72 (2005) 035009.

[32] H. Baer, H. Prosper and H. Summy, Phys. Rev. D 77 (2008) 055017.

[33] J. Ellis, J. Hagelin, D. V. Nanopoulos and M. Srednicki. Phys. Lett. B 127 (1983) 233.

[34] F. Gianotti and M. L. Mangano, hep-ph/0504221; H. Baer, V. Barger and G. Shaughnessy, arXiv:0806.3745 (2008).

[35] R. M. Barnett, J. Gunion and H. Haber, Phys. Lett. B 315 (1993) 349; H. Baer, X. Tata and J. Woodside, Phys. Rev. D 41 (1990) 906 and Phys. Rev. D 45 (1992) 142.

[36] H. Baer, K. Hagiwara and X. Tata, Phys. Rev. D 35 (1987) 1598; H. Baer, D. Dzialo-Karatas and X. Tata, Phys. Rev. D 42 (1990) 2259; H. Baer, C. Kao and X. Tata, Phys. Rev. D 48 (1993) 5175; I. Hinchliffe, F. Paige, M. Shapiro, J. Soderqvist and W. Yao, Phys. Rev. D 55 (1997) 5520. 
[37] H. Baer, M. Drees, F. Paige, P. Quintana and X. Tata, Phys. Rev. D 61 (2000) 095007.

[38] H. Bachacou, I. Hichliffe, F. E. Paige, Phys. Rev. D 62 (2000) 015009; B.K. Gjelsten, E. Lytken, D.J. Miller, P. Osland and G. Polesello, ATLAS internal note ATL-PHYS-2004-007 (2004), published in: G. Weiglein et al. [LHC/LC Study Group], Phys. Rept. 426 (2006) 47; M. M. Nojiri, G. Polesello, D. Tovey, J. High Energy Phys. 0805 (2008) 014; CMS Collaboration (G.L. Bayatian et al.), CMS technical design report, volume II: Physics performance, CERN-LHCC-2006-021, CMS-TDR-008-2, J. Phys. G 34 (2007) 995.

[39] H. Baer, V. Barger, G. Shaughnessy and H. Summy, Phys. Rev. D 75 (2007) 095010.

[40] H. Baer, C. H. Chen, F. Paige and X. Tata, Phys. Rev. D 53 (1996) 6241.

[41] H. Baer, T. Krupovnickas, S. Profumo and P. Ullio, J. High Energy Phys. 0510 (2005) 020.

[42] W. F. Long and T. Stelzer, Comput. Phys. Commun. 81 (1994) 357. 